\title{
TREM2 Limits Progression of Deficits and Spreading of Tau Pathology in Mice
}

\section{Astrid F. Feiten}

Macquarie University

Carol Au

Macquarie University

Annika van Hummel

Macquarie University

Julia van der Hoven

Macquarie University

Yuanyuan Deng

Macquarie University

Magdalena Przybyla

Macquarie University

Fiona Bright

Macquarie University

\section{Troy Butler}

Macquarie University

\section{Fabien Delerue}

Macquarie University

\section{Amer Toutonji}

Medical University of South Carolina

\section{Silvia Guglietta}

Medical University of South Carolina

\section{Susanne Wegmann}

German Centre for Neurodegenerative Diseases (DZNE)

\section{Bradley T. Hyman}

Massachusetts General Hospital

\section{Carsten Krieg}

Medical University of South Carolina

\section{Yazi D. Ke}

Macquarie University

Lars Ittner ( $\nabla$ lars.ittner@mq.edu.au )

Macquarie University https://orcid.org/0000-0001-6738-3825 
Research article

Keywords: TREM2, tau, spreading, dementia, Alzheimer's disease, microglia signature, microglia activation, neuroinflammation

Posted Date: August 2nd, 2021

DOl: https://doi.org/10.21203/rs.3.rs-741896/v1

License: (c) (i) This work is licensed under a Creative Commons Attribution 4.0 International License.

Read Full License 
4 Przybyla ${ }^{1}$, Fiona Bright ${ }^{1}$, Troy Butler $^{1}$, Fabien Delerue ${ }^{1}$, Amer Toutonji, ${ }^{2}$ Silvia Guglietta, ${ }^{2}$ Susanne Wegmann ${ }^{3}$,

$7 \quad{ }^{1}$ Dementia Research Centre and Department of Biomedical Sciences, Faculty of Medicine, Health and Human

8 sciences, Macquarie University, Sydney, NSW 2109, Australia

$9 \quad{ }^{2}$ Hollings Cancer Center, Department of Microbiology \& Immunology, Medical University of South Carolina

10 (MUSC), Charleston, SC 29425, United States.

$11{ }^{3}$ German Centre for Neurodegenerative Diseases (DZNE), 10117 Berlin, Germany

$12{ }^{4}$ Massachusetts General Hospital, Harvard Medical School, Charlestown, MA 02129, United States.

$13{ }^{ \pm}$Corresponding author

14 AF Feiten, astrid-feentje.feiten@hdr.mq.edu.au; C Au, carol.au@mq.edu.au; A van Hummel, 15 annika.vanhummel@mq.edu.edu.au; J van der Hoven, julia.vanderhoven@hdr.mq.edu.au; Y Deng,

16 yuanyuan.deng1@hdr.mq.edu.au; M Przybyla, magdalena.przybyla@mq.edu.au; F Bright,

17 fiona.bright@mq.edu.au; T Butler, troy.butler@mq.edu.au; F Delerue, fabien.delerue@mq.edu.au; A Toutonji, toutonji@musc.edu; S Guglietta, gugliett@musc.edu; S Wegmann, susanne.wegmann@dzne.de; BT Hyman,

19 bhyman@mgh.harvard.edu; C Krieg, carsten.krieg@gmail.com; YD Ke, yazi.ke@mq.edu.au; LM Ittner, lars.ittner@mq.edu.au

\section{Abstract}

Background. Amyloid- $\beta(\mathrm{A} \beta)$ and tau form pathogenic lesions in Alzheimer's disease (AD) brains. As AD clinically progresses, tau pathology propagates in a very distinct pattern between connected brain areas. The molecular mechanisms underlying this tau pathology spread remain largely unknown. Genome-wide association studies have identified polymorphisms in triggering receptor expressed on myeloid cells 2 (TREM2) as genetic risk factors for $\mathrm{AD}$ and regulators of $\mathrm{A} \beta$ pathology-dependent tau propagation. Whether TREM2 contributes to neuron-to-neuron spreading of pathological tau remains unknown. 
Methods. Here, we crossed Trem2-deficient mice with P301S tau transgenic TAU58 mice and subjected the

30

31

32 mice to behavioral testing and assessed neuropathology. Microglial activation states were determined using cytometry by of flight (CyTOF) and quantitative PCR. Tau spreading was assessed in vivo using tracing of focal tau expression.

Results. Trem 2 depletion significantly aggravated tau-induced early-onset motor and behavioural deficits. Neuropathologically, Trem 2 reduction increased the number of hyperphosphorylated tau lesions in young TAU58 brains and reduced disease-associated microglia. Direct assessment of inter-neuronal spread of tau in vivo revealed significantly enhanced propagation of tau in the absence of Trem2, suggesting that microglial TREM2 limits the progression of tau pathology in disease.

Conclusion. Taken together, our data suggests that reduced TREM2 function accelerates the onset and progression of functional deficits and tau neuropathology in tau transgenic mice, which is - at least in part - due to increased tau spreading. Therefore, reduced TREM2 function may contribute to early AD by augmenting tau toxicity and its inter-neuronal propagation.

\section{Keywords}

TREM2; tau; spreading; dementia; Alzheimer’s disease; microglia signature; microglia activation; neuroinflammation

\section{Background}

Alzheimer's Disease (AD) is the most prevalent form of dementia $(1,2)$. Cognitive decline is often accompanied by behavioural changes, anxiety, depression or sleep disturbances, together leading to full-time care dependency and ultimately death $(3,4)$. AD brains are characterised by two pathological hallmark changes, the extracellular deposition of amyloid- $\beta(A \beta)$ in plaques and the formation of intracellular neurofibrillary tangles (NFT), composed of hyper phosphorylated microtubule-associated protein tau (5). Fibrillary tau pathology (without A $\beta$ plaques) is furthermore characterizing brains in different forms of frontotemporal dementia (FTD), the second most common form of dementia in individuals age 65 or younger (6) and coining the term 'tauopathies'. Studies into familial FTD identified pathogenic mutations in the tau encoding MAPT gene, highlighting its role in 

(9).

Over 25 years ago, Braak \& Braak showed that NFT deposition in AD brains occurs in a highly conserved pattern that correlates with disease progression (10). Accordingly, tau pathology spreads in a stereotypical fashion, occurring first in entorhinal areas before affecting connected limbic and cortical brain areas (10). The molecular and cellular mechanisms that drive tau propagation remain largely unknown (11). Mouse models recapitulating the distribution pattern have been used to show neuron-to-neuron spread of tau, most likely via synaptic connections (12), inducing recipient neuron tau to aggregate (11). Further, in has been shown that extracellular tau aggregates can be taken up by cells and induce tau aggregation in culture (13). More recently, microglial involvement in tau propagation has emerged (14-16), however the exact mechanisms remain to be defined. It has previously been shown that microglia can internalize tau in vitro and in vivo (17) and degrade hyperphosphorylated tau (18). Interestingly, several polymorphisms of microglial genes have been linked to increased AD risk over the past years (reviewed in $(19,20))$. Accordingly, genome wide association studies identified polymorphisms of the gene triggering receptor expressed on myeloid cells 2 (TREM2) linked to a higher risk to develop AD (21). The most common variant, $\mathrm{R} 47 \mathrm{H}$, conveys a 3-4 fold risk increase for late onset $\mathrm{AD}$ (LOAD) $(21,22)$. While it is not fully understood how TREM2 mutations increase the risk for $\mathrm{AD}$, loss of function rather than a toxic gain of function has been proposed (21). Subsequent studies found additional polymorphisms and an association with an increased risk for FTD $(23,24)$ and other neurodegenerative diseases like amyotrophic lateral sclerosis (ALS) $(25,26)$ or Parkinson's disease $(27,28)$. TREM2 is a transmembrane receptor of the immunoglobulin superfamily and predominately expressed by microglia in the brain. TREM2 signalling has been linked to different cellular processes of innate immunity including phagocytosis $(29,30)$, proliferation $(31)$, promotion of survival $(32,33)$ as well as the secretion of cytokines and chemokines (34). Activity of TREM2 has been reported to be involved in the clearance of apoptotic neurons (29). Further, TREM2 is a receptor for lipoproteins such as APOE (35) and can bind $A \beta(36,37)$. Consequently, early studies have focused on TREM2 and A $\beta(38-45)$, while its link to tau was only more recently investigated (46-50). Apparently contradicting findings with TREM2 and A $\beta(38-44)$, were likely due to differential roles of TREM2 during disease stages (45). Similarly, early TREM2-tau literature was somewhat contradictive; while some reported that Trem 2 depletion attenuated neurodegeneration in tau transgenic mice $(47,48)$, others found detrimental effects of Trem 2 loss $(46,49,50)$ which now is the emerging consensus. Augmented spatial learning deficits in tau transgenic mice upon hippocampal shRNA-mediated 
TREM2 reduction has been observed (49), however, a detailed functional analysis of Trem2-deficient tau transgenic mice has not been done to date. Furthermore, the effects of reduced TREM2 function on tau propagation has not been assessed.

In the present study, we crossed the established tau P301S transgenic line TAU58 (51-54) with Trem2knockout mice, generated by CRISPR/Cas9-mediated gene targeting. We found that TREM2 reduction worsened motor deficits of TAU58 mice and aggravated behavioural deficits together with accelerated tau neuropathology in young but not older mice. Interestingly, numbers of active, disease-associated (vs. resting/homeostatic) microglia were reduced early-on in TREM2-deficient TAU58 mice. Injecting mice with an adeno-associated virus $(\mathrm{AAV})$ to visualize tau protein spread between neurons $(55,56)$ revealed increased tau propagation throughout the brains of TREM2 depleted mice. Our data supports a disease-limiting role of TREM2 in onset and propagation of tau pathology.

\section{Methods}

Mice All animal experiments were approved by the Animal Ethics Committees of Macquarie University. Mice were group housed on a normal $12 \mathrm{~h}$ light/dark cycle and had access to food and water ad libitum. TAU58 (TAU58/2 line) transgenic mice express the human P301S mutation on the 0N4R tau isoform under the control of the murine Thy 1.2 neuronal promoter on a C57B1/6 background and have been described previously (51-53). The first exon of the murine Trem2 gene (ENSEMBL ENSMUSG00000023992) was targeted to remove the endogenous start codon (Figure S1A) by CRISPR-mediated gene targeting using two guides (Guide 1: 5'TGCACAAGGTCCCCTCCGGC-3'; Guide 6: 5'-CTGAAGGTAGGTCCCTCCTG-3'). These single-guide RNAs (sgRNAs) were rationally designed using a computational tool to minimize off-targets (http://crispr.mit.edu) and were produced using a non-cloning method whereby a T7-conjugated forward primer generates a linear template by PCR, as previously described (57). Briefly, the sgRNA scaffold of the pX330 (Addgene \#42230, gift from Dr. Feng Zhang) was used to synthesize a small linear DNA template. Next, this template was in-vitro transcribed using a T7 Quick High Yield RNA synthesis kit (NEB \#E2050S) following the manufacturer's instructions. The resulting sgRNAs were purified using NucAway Spin columns (ThermoFisher \#AM10070) and incubated with S.p.Cas9 protein (NEB \#M0646T) to form ribonucleoprotein complexes (RNPs). RNPs were electroporated (NEPA21, Nepagene) into fertilised C57Bl/6 zygotes with the respective concentrations: 200ng/ul Cas9, 300ng/ul Guide 1 and 150ng/ul Guide 6. Nineteen live pups were produced, nine 
of which displayed a deletion at the endogenous Trem 2 locus by PCR genotyping (Primer sequences: Fwd 5'TAAGAAGCCACTGTCCTCCAATCT-3'; Rev 5'-AGAAAAGCAGAGTCAAAGCAGGAC-3'). One founder was selected to establish the colony based on Sanger sequencing (Macrogen, South Korea) of the edited allele (Figure S1B), which revealed a 92bp deletion including the start codon. Homozygous $(\Delta / \Delta)$ and heterozygous $(\Delta /+)$ Trem $2 \mathrm{KO}$ mice were identified via PCR using the above genotyping primers (Figure S1C). Trem $2 \mathrm{KO}$ mice were maintained on a C57B1/6 background and crossed with TAU58 transgenic mice to generate Trem2 heterozygous TAU58 transgenic (TAU58/Trem $2^{4 /+}$ ) and $\operatorname{Trem} 2$ heterozygous $\left(\operatorname{Trem} 2^{4 /+}\right.$ ) mice. These mice were used as breeders to create the experimental mice, including all littermate controls (Figure 1B). Animals were subjected to behavioural test battery at 1-, 2-, 3- and 6 months of age. Both genders were used, and different animals were used at each time point, to avoid potential artefacts induced by re-testing mice on the same test. Group sizes for each test are provided in Table S1.

RotaRod To assess motor function mice were placed on the RotaRod (UgoBasile, Gemonio VA, Italy) as described elsewhere (58). Briefly, mice were placed on a rotating rod with accelerating speed (acceleration mode 5-60rpm over 120 seconds). The latency to fall or until mice did one full revolution was recorded. Mice were trained for 3 consecutive days, 5 trials each day and the best trial of the last day was used for analysis.

Beam To further assess the fine-motor performance, mice were subjected to the Beam traversing challenge (51). For this challenge mice need to cross a thin elevated wooden beam. The house of their home cage was placed at the end of the beam, on a platform the same height as the beam. Mice were trained in the morning and tested in the afternoon of the same day. The testing session was recorded, and mice performed two consecutive trials. The videos were analysed manually, by an experimenter blinded to the experimental groups. The time to cross as well as the number of foot slips was counted. The two trials were averaged for analysis. If mice fell off the beam in one trial, the successful trial was used for analysis. Mice that fell in both trials were excluded from the analysis.

Morris Water Maze To assess spatial memory, mice were subjected to Morris Water Maze (MWM) testing (59). The test apparatus consisted of a large tank (1.4 m diameter), a Perspex platform $(10 \mathrm{~cm}$ diameter, $40 \mathrm{~cm}$ high) and four different visual cues. Each visual cue was placed equidistant from one another around the tank. The platform was positioned roughly $20 \mathrm{~cm}$ from the inside wall of the tank. The tank was filled with water to a height of approximately $0.5-1 \mathrm{~cm}$ above the platform with white non-toxic acrylic-based paint diluted in the water to conceal the platform. Additionally, the lights in the testing room were dimmed. Mice were acclimatised 
in the room for one hour prior to testing. On days 1-5, mice were trained to find the hidden platform. To achieve

147 this, mice had 4 swims per day, each trial starting from a different position. This was done to ensure mice

148 learned the location of the platform rather than the swimming trajectory. Additionally, the order of the positions

149 was changed every day. Mice were given 1 minute to locate the platform and then had to sit on the platform for

150 one additional minute once they found it. When mice did not find the platform within 1 minute they were guided

151 to it and had to stay on the platform for a minute. Two to three mice were alternated per round, to avoid fatigue

152 of the mice. The order of the mice and the number per round was kept consistent for the whole duration of the test. Each trial was recorded and the latency to find the platform was noted. On day 6 mice were subjected to the probe trial for 30 seconds. The hidden platform was removed from the maze and mice were placed into the tank from position 1 and recorded. The video was analysed using AnyMAZE software (Stoelting). For the analysis, the tank was divided into four quadrants (the target quadrant, the right quadrant, the left quadrant and the opposite quadrant) and the platform zone (a circle corresponding to the size and position of the platform). The software counted the number of entries into the platform zone, the latency for the first entry into the platform zone, the time spent in the platform zone as well as the time spend in each of the quadrants and the overall swim speed and distance.

A visual cue test was performed after the probe trial to ensure all mice used have sensorimotor abilities and motivation. The platform was placed back into the same position as before, with a flag placed on top of it. Mice had four swims from positions 1 through 4 . The latency to reach the platform/ flag was noted and mice were taken out of the maze as soon as they reached the platform. The four trials were averaged and an exclusion criteria of an average time greater than 20 seconds was applied.

Elevated Plus Maze To test for disinhibition behaviour mice were subjected to Elevated Plus Maze (UgoBasile, Gemonio VA, Italy; EPM) testing at all ages. The EPM consist of two open and two closed arms $(35 \mathrm{~cm} \times 5.5 \mathrm{~cm}$ each) and an inner area where all four arms meet $(5.5 \mathrm{~cm} \times 5.5 \mathrm{~cm})$ and is elevated $60 \mathrm{~cm}$ off the ground. Mice were acclimatised in the room for one hour prior to testing. At the start of the test mice were placed in the inner area, facing the open arm, and allowed to explore for $5 \mathrm{~min}$ while being recorded. The time mice spend in the different areas was analysed using the AnyMAZE software (Stoelting).

Open Field Activity and exploration behaviour was assessed using the Open Field test (OF), as described previously (54). Briefly, the OF consists of an open arena $(40 \mathrm{~cm} \mathrm{x} 40 \mathrm{~cm})$ placed in an enclosed space containing a camera as well as a ceiling-mounted fan. Mice were acclimatised in the testing space for an hour prior to the test. At the start of the test, mice were placed in the top right corner of the arena and were allowed to 
explore it freely for 10 minutes. Mice were video recorded while performing the test and the videos were later analysed using AnyMAZE software (Stoelting). For the analysis, the arena was divided into an inner zone (17.5 $\mathrm{cm} \times 17.5 \mathrm{~cm}$ square in the middle) and an outer zone. The software tracked how much time animals spent in each respective zone as well as the total distance travelled.

AAV production Packaging of recombinant AAVs with AAV1 capsid (Penn Vector Core - Gene Therapy Program, University of Pennsylvania) was performed as previously described (60). AAV1-eGFP-2a-P301L-tau plasmid was a gift from Bradley Hyman $(55,56)$. Titres of AAVs were determined by quantitative polymerase chain reaction (qPCR) and liquid droplet PCR using the following primers (WPRE_F: ggctgttgggcactgacaat and WPRE_R: ccgaagggacgtagcagaag).

Stereotaxic Injections 4-week-old mice were anaesthetised with 7.2 $\mu 1 / \mathrm{g}$ of Ketamine/Xylazine. Their heads were fixed in a stereotaxic frame (Kopf instruments). A small midline incision was made and bregma located. A small burr hole was made above the entorhinal cortex (Coordinates: A/P: 3; M/L: -3 both from bregma and $\mathrm{D} / \mathrm{V}$ : -3 from skull surface) using a bone micro-drill (Fine Science Tools). $1 \mu$ l of AAV (titre: $1.64 \times 10^{13}$ viral genomes) was injected at a rate of $300 \mu \mathrm{l} /$ minute. The needle was left in place for 5 minutes to reduce backflow after injection.

Histological Analysis Mice were anaesthetised and transcardially perfused with ice-cold phosphate buffered saline (p.H 7.4). Brains were carefully removed and separated into hemispheres. The right hemisphere was further sub-dissected into hippocampus and cortex, snap frozen in liquid nitrogen and stored at $-80^{\circ} \mathrm{C}$ for biochemical analysis. The left hemisphere was used for immunohistochemical analysis which was carried out as described previously (61). Mice that had undergone stereotaxic surgery were additionally perfused with $4 \%$ paraformaldehyde prior to removal of the brain and the whole brain was used for immunohistochemistry. Briefly, the brain was post-fixed in cold $4 \%$ paraformaldehyde overnight and changed to $70 \%$ ethanol the next day. Tissue was placed in histology cassettes and processed overnight in the Excelsior tissue processor (ThermoFisher, Waltham, MA, USA). Processed tissue was embedded in paraffin and sectioned either coronally at $3 \mu \mathrm{m}$ or horizontally at $5 \mu \mathrm{m}$ (whole brain). Sections were allowed to air dry over night before being baked at $65^{\circ} \mathrm{C}$ for two hours. To remove any residual paraffin, slides were placed in xylene for 20 minutes and then rehydrated by immersing the slides in decreasing concentrations of ethanol. Heat-mediated antigen retrieval was performed in a Milestone Histology microwave (Milestone, Sorisole, Italy) using citrate buffer. Sections were 
buffer (BB, containing 3\% heat inactivated Goat Serum 2\% Bovine Serum Albumin in PBS) for one hour at room temperature. $100 \mu 1$ primary antibody diluted in $\mathrm{BB}$ was added and slides were incubated overnight at $4{ }^{\circ} \mathrm{C}$ (Table S2). The next day slides were washed with PBS. AlexaFluor (Invitrogen, Carlsbad, California, United States) conjugated secondary antibody (Table S2) and DAPI (1:1000, Molecular Probes, Carlsbad, Ca, USA) were diluted in $\mathrm{BB}$, and $100 \mu 1$ were added on to the slides and incubated for one hour at room temperature. Slides were washed and mounted with Fluoromount-G (SouthernBiotech, Birmingham, Alabama, United States) mounting media. Slides were scanned using an Axioscan Z.1 slide scanner (Zeiss, Oberkochen, Germany). Quantification of images was performed using either the Zeiss ZEN 2.6 blue edition software or ImageJ. Inkscape was used to create the figures and construct the heat maps. For the heat maps counts were divided into 14 different areas and averaged across mice per genotype, per depth. Depth is given relative to bregma.

RNA Extraction. Mice for RNA extraction were sacrificed, brains were removed and sub-dissected into hippocampus and cortex. Tissues were snap frozen in liquid nitrogen. RNA was extracted according to manufacturer's protocol for TRIzol (ThermoFisher Scientific). Briefly, tissue was homogenised in TRIzol using a plastic pestle. Lysates were centrifuged briefly to remove debris. Chloroform was added to the supernatant to precipitate proteins. After a short incubation period, tubes were centrifuged to separate the aqueous from the phenol phase. The aqueous phase was transferred to a new tube and 70\% ethanol added. After gentle mixing, RNA purification was performed by using the Qiagen RNeasy Kit according to manufacturer's instructions, including the optional DNase treatment. RNA concentrations were measured using a Nanodrop (ThermoFisher Scientific).

qRT-PCR First-strand cDNA was synthesised from the extracted RNA by using the SuperScript VILO MasterMix (ThermoFisher Scientific) according to manufacturer's instructions. $1 \mu \mathrm{g}$ of RNA was used in the cDNA reaction and resulting cDNA was diluted 1:10 for qRT-PCR. Standards were made from pooled samples. A master mix consisting of 2x Power SYBR Green (ThermoFisher Scientific), DEPC treated water and the respective primers was made for each gene of interest for qRT-PCR analysis. Primers were designed specifically for qRT-PCR and ordered from Macrogen (South Korea) (Primer list Table S3). qRT-PCR was run on a ViiA 7 Real-Time PCR system (ThermoFisher Scientific).

Brain single cell suspensions. Following perfusion with 1X PBS, brains from adult mice were collected and placed in ice-cold Hibernate A medium (A1247501, Thermo Fisher Scientific). The brain was transferred to a 
petri dish on ice without media and cut into $2 \mathrm{~mm}^{2}$ pieces with a scalpel blade. Tissue dissociation was performed with Digestion Media containing $1 \mathrm{mg} / \mathrm{mL}$ Collagenase D (Sigma) dissolved in Hibernate A Medium in a GentleMACS C tube (Miltenyi Biotec). The tissue was incubated at $37^{\circ} \mathrm{C}$ for 30 min with stirring using program 37C_ABDK_01 on the GentleMACS Octo Dissociator (Miltenyi Biotec). Tissue suspensions were further mechanically dissociated by gentle trituration using a $5 \mathrm{~mL}$ serological pipette for twenty strokes. The cell suspension was then passed through a pre-wetted $70 \mu \mathrm{m}$ cell strainer with a syringe plunger and washed with ice-cold $1 \mathrm{X}$ PBS without calcium and magnesium. After centrifugation at $600 \mathrm{xg}$ for 4 min at $4{ }^{\circ} \mathrm{C}$, the supernatant was discarded and the cell pellet resuspended in $10 \mathrm{~mL}$ of $1 \mathrm{X}$ PBS. The cell suspension was transferred to a $15 \mathrm{~mL}$ conical tube and mixed with $5 \mathrm{~mL}$ of isotonic Percoll (9 parts Percoll: 1 part 10X PBS) for a final $30 \%$ (vol/vol) Percoll gradient. This was centrifuged at $1500 \mathrm{x} \mathrm{g}$ for $20 \mathrm{~min}$ at $4{ }^{\circ} \mathrm{C}$ with maximum acceleration and no brakes during deceleration. The cloudy white myelin overlay was removed by aspiration, and the remaining supernatant and cell pellet were resuspended and transferred to a new $15 \mathrm{~mL}$ conical tube. The cells were centrifuged at $600 \mathrm{x} g$ for $4 \mathrm{~min}$ at $4^{\circ} \mathrm{C}$, the supernatant was discarded and the cell pellet resuspended in $200 \mu \mathrm{L}$ FACS Buffer (2\% FBS, 2mM EDTA, 1X PBS). A hemocytometer was used to confirm single cell dissociation and viability before proceeding with cell barcoding and staining.

Mass cytometry. Single cell suspensions from mouse brains were Live/Dead stained with Cisplatin-198 for three minutes at room temperature. To achieve increased throughput and homogenous staining, metal cell barcoding against mouse CD45 positive cells was used (62-64). The metal isotopes $106 \mathrm{Cd}, 110 \mathrm{Cd}, 111 \mathrm{Cd}$, $112 \mathrm{Cd}, 113 \mathrm{Cd}, 114 \mathrm{Cd}$, and $116 \mathrm{Cd}$ were conjugated to anti-mouse CD45.2 antibodies according to manufacturer's instruction using the MCP9 Antibody Labeling kit (Fluidigm). A seven choose three barcoding matrix was generated and barcoding reagents were titrated to achieve optimal labelling. Cells were stained in 96-well plates. To each sample a unique combination of exactly three metal cell barcoding reagents diluted in 50uL PBS, 0.5\% BSA was added and then incubated for $20 \mathrm{~min}$ at room temperature. Cells were washed with $150 \mathrm{uL}$ FACS buffer at $4^{\circ} \mathrm{C}$. Barcoded cells were then combined in a single $1.5 \mathrm{ml}$ tube and washed with FACS buffer. Surface staining mix was prepared by diluting all primary antibodies in FACS buffer. Cell suspension was centrifuged at $600 \mathrm{~g}$ for four minutes at $8^{\circ} \mathrm{C}$ and the supernatant was removed. Cells were re-suspended in surface stain mix (Table S4) and incubated at room temperature for 20 minutes. Cells were washed in FACS buffer and staining was repeated with secondary antibodies at room temperature for 20 minutes. Cells were washed in FACs buffer and incubated over night with 250nM iridium intercalator (Fluidigm) in Maxpar cell fix./perm. buffer (Fluidigm) to label cellular DNA. Subsequently, cells were washed with PBS followed by 

performed on a CyTOF2.1 (Helios) mass cytometer (Fluidigm). After acquisition, data was normalized by using the bead standard and the executable MATLAB normalizer application, dead cells and beads removed and debarcoded using Boolean gating using FlowJo software (BD) (65). All analyses on CyTOF data were performed after arcsinh (with cofactor equal to 5) transformation of marker expression. Data was analyzed in R using our published bioinformatic workflow (66).

Statistical analysis Statistical analysis was performed using GraphPad Prism software (GraphPad, La Jolla, CA, USA). A value of $\mathrm{p}=0.05$ was considered significant. One-way analysis of variance (ANOVA) tests was used to compare more than two groups and two-way ANOVA's were used to compare groups across time. Students ttest was performed to compare two groups.

\section{Results}

Accelerated deficits in P301S tau transgenic mice upon Trem 2 reduction. To study the role of $\operatorname{Trem} 2$ in tau pathology in vivo, we generated a new Trem2-deficient mouse line, $\operatorname{Trem} 2^{4 / 4}$, by introducing a 92 base pair deletion in exon 1 of Trem 2 comprising its translational start codon using CRISPR/Cas9-mediated genome editing in C57Bl/6 mice (Figure S1A). Quantitative PCR revealed half the Trem 2 mRNA levels of wild-type C57Bl/6 mice in the brains of heterozygous Trem $2^{4 /+}$ mice, while no Trem 2 mRNA was detectable in homozygous $\operatorname{Trem} 2^{1 / 4}$ mice (Figure 1A). To determine whether Trem 2 modulates functional deficits of tau transgenic mice, we crossed TAU58 mice expressing neuronal human P301S mutant tau with the Trem $2^{4 / 4}$ strain to obtain TAU58/Trem $2^{4 / 4}$, TAU58/Trem $2^{4 /+}$ and TAU58/Trem $2^{+/+}$mice (Figure 1B) and subjected them to functional testing at different ages. Unless otherwise specified, littermate controls refer to $\operatorname{Trem} 2^{4 / 4}, \operatorname{Trem} 2^{1 /+}$ and $\mathrm{Trem}_{2}{ }^{+/+}$mice that do not carry the P301S tau transgene. We have previously reported learning deficits in 4-month-old TAU58 mice when subjected to Morris water maze (MWM) testing for spatial memory formation (53). To study mice at pre-symptomatic stage, we tested young 3-month-old mice and found no learning performance deficits in TAU58/Trem $2^{+/+}$and TAU58/Trem $2^{1 /+}$ compared to Trem $2^{+/+}, \operatorname{Trem} 2^{1 /+}$ and $\operatorname{Trem} 2^{1 / 4}$ littermate controls (Figure 1C). In contrast, 3-month-old TAU58/Trem $2^{1 / 4}$ mice displayed significantly delayed learning in the MWM acquisition phase while the probe trial was unaffected. We have furthermore previously reported motor deficits in 3-month-old TAU58 mice, which were phenotypically still normal at 2 months of age $(51,52)$. Accordingly, TAU58/Trem $2^{+/+}$mice performed like littermate controls during Rotarod testing at 1 and 
2 months of age (Figure 1D). While TAU58/Trem $2^{4 /+}$ and TAU58/Trem $2^{4 / 4}$ mice performed normally at 1

294

295

296

297

298

299

300

301

302

303

304

305

306

307

308

309

310

311

312

313

314

315

316

317

318

319

320

321

322 month of age, they presented significant deficits as compared with littermate controls at 2 months of age, with TAU58/Trem $2^{4 / 4}$ mice significantly impaired compared to TAU58/Trem $2^{+/+}$mice. At 3 months of age, TAU58/Trem $2^{+/+}$have deteriorated and presented similar deficits as TAU58/Trem $2^{4 /+}$ and TAU58/Trem $2^{4 / 4}$ mice compared to littermates. These deficits progressed further by 6 months of age. We have previously shown that TAU58 mice present with increased foot slips while crossing a narrow beam at 3 months of age (51). In the present cohort, significant differences in the number of foot slips began in 1 month-old TAU58/Trem $2^{4 /+}$ mice, which progressed to include TAU58/Trem $2^{\mathrm{A} / 4}$ mice by 2 and 3 months of age (Figure 1E). No significant differences in the number of foot slips between the three genotypes was detected in the 6 months cohort. Analysing the time taken to cross the narrow beam, we found significant differences only in 1 month-old TAU58/Trem $2^{4 /+}$ mice compared to TAU58/Trem $2^{+/+}$mice, with TAU58/Trem $2^{4 /+}$ mice taking longer to cross the beam (Figure 1E). The analysis of the 3- and 6-month-old cohorts remained incomplete as a large number of transgenic mice fell of the beam during testing. Notably, $16 \%$ of 3-month-old TAU58/Trem $2^{4 / 4}$ and TAU58/Trem $2^{4 /+}$ mice fell off the beam during both trials, while all TAU58/Trem $2^{+/+}$mice completed at least one trial successfully. $40 \%$ of 6 months-old TAU58/Trem $2^{4 / 4}$ mice fell off the beam during both trials and an additional $20 \%$ fell during one of the trials, while $66 \%$ of TAU58/Trem $2^{4 /+}$ mice fell during both and $22 \%$ during one of the trials compared to $30 \%$ (both trials) and $20 \%$ (one trial) of TAU58/Trem $2^{+/+}$mice (Figure 1F). In addition to motor deficits, TAU58 mice present with an early-onset and progressive disinhibition behaviour that presents as increased time mice spent in the open arms of the Elevated Plus Maze (EPM) $(52,67)$. TAU58/Trem $2^{+/+}$, TAU58/Trem $2^{4 /+}$ and TAU58/Trem $2^{4 / 4}$ mice showed trends toward increased open arm times compared to littermate controls at 1 month of age (Figure 1G). By 2-month-old, TAU58/Trem $2^{4 / \Delta}$ mice progressed to significant deficits as compared with TAU58/Trem $2^{+/+}$littermates. Interestingly, 6-month-old $\operatorname{Trem} 2^{4 / 4}$ mice displayed significantly increased open arm time and activity compared to $\operatorname{Trem} 2^{1 /+}$ or $\operatorname{Trem} 2^{+/+}$ mice. Overall locomotor activity was comparable in EPM (Figure S2A), as well as in the open field for all groups and ages tested (Figure S2B). Taken together, Trem2 reduction in TAU58 mice resulted in earlier onset of learning, motor and behavioural deficits. Interestingly, Trem 2 knockout appeared to have disinhibitory effects independent of transgenic tau expression as mice aged.

Increased tau phosphorylation upon Trem 2 loss in young TAU58 mice. TAU58 mice are characterized by progressive tau hyperphosphorylation with brain region-specific differences in onset and progression of 

process, while phosphorylation at serine 422 (pS422) is described to be present later in disease course (68). We have previously reported the presence of tau pS214 in TAU58 amygdala as young as 3 months of age with the late-stage marker tau pS422 appearing from 6 months of age (52). Here, we found that pS214 occurred in the amygdala as early as 1 month of age (Figure 2A). By immunostaining of brain sections, there was no notable difference in levels of pS214 between TAU58/Trem $2^{+/+}$, TAU58/Trem $2^{4 /+}$ and TAU58/Trem $2^{4 / 4}$ mice. For comparison, pS214 showed a similar pattern in the motor cortex (Figure S3A). pS422 labelling was sparse at 1 and 2 months of age with no differences between TAU58/Trem $2^{+/+}$, TAU58/Trem $2^{4 /+}$ and TAU58/Trem $2^{4 / 4}$ mice (Figure 2B). However, at 3 months of age, TAU58/Trem $2^{4 / 4}$ mice showed significantly more pS422 positive cells in the amygdala as compared with TAU58/Trem $2^{+/+}$and TAU58/Trem $2^{4 /+}$ mice. We observed a similar trend towards increased numbers of pS422-positive cells in the motor cortex of 2-months-old TAU58/Trem $2^{1 /+}$ and TAU58/Trem $2^{4 / 4}$ mice (Figure S3B). At 6 months of age, levels of pS422 were comparably high in TAU58/Trem $2^{+/+}$, TAU58/Trem $2^{4 /+}$ and TAU58/Trem $2^{4 / 4}$ mice, consistent with our previously reported numbers (52). Taken together, Trem2 depletion in TAU58 mice resulted in accelerated tau phosphorylation at the late-stage disease site pS422.

Trem 2 reduction reduces tau-dependent microglial activation. Given that microglial activation associated with neurodegenerative processes is regulated by Trem2 (69), we next determined microglial homeostasis in TAU58/Trem $2^{+/+}$, TAU58/Trem $2^{4 /+}$ and TAU58/Trem $2^{4 / 4}$ mice. Staining of brain sections with the microglial marker Iba1 revealed no overt differences in distribution and morphology of microglia between Trem $2^{+/+}$, $\operatorname{Trem} 2^{4 /+}$ and $\operatorname{Trem} 2^{\Delta / \Delta}$ mice, or between TAU58/Trem $2^{+/+}$, TAU58/Trem $2^{4 /+}$ and TAU58/Trem $2^{4 / \Delta}$ mice (Figure S3C). In all TAU58 lines, microglia were frequently observed in the proximity of neurons with transgenic P301S tau expression (Figure 3A), consistent with our previous reports of microglial engulfment of neurons harbouring tau pathology (51). To obtain quantitative data on microglial populations in TAU58/Trem $2^{+/+}$, TAU58/Trem $2^{4 /+}$ and TAU58/Trem $2^{4 / \Delta}$ mice and the respective littermate controls, we obtained single cells from brains of TAU58/Trem $2^{+/+}$, TAU58/Trem $2^{4 /+}$ and TAU58/Trem $2^{4 / \Delta}$ mice and subjected them to cytometry by time of flight (CyTOF) analysis with a panel of microglial markers (Table S4). This panel includes markers to differentiate between distinct populations of resting state and activated microglia in all genotypes (Figure 3B and 3C). Elevated numbers of activated microglia were observed in TAU58/Trem $2^{+/+}$and TAU58/Trem $2^{4 /+}$ mice with a concomitant reduction of resting state microglia (Figure 
353 3D). In contrast, TAU58/Trem $2^{4 / \Delta}$ exhibited numbers of resting state and activated microglia comparable to

354 Trem $2^{+/+}$, Trem $2^{4 /+}$ and Trem $2^{4 / \Delta}$ mice, suggesting the absence of overt microglia activation. Supporting these

355 findings, quantitative PCR showed increased mRNA levels of selected microglial activation markers (CD16,

356 APOE) and a converse reduction of homeostatic marker genes (Siglech) in TAU58/Trem2 $2^{+/+}$brains compared to

357 TAU58/Trem $2^{4 /+}$, and to an even higher degree compared to TAU58/Trem $2^{4 / 4}$ littermates (Figure 3E). For

358 example, CD16, a surface marker of M1 activated microglia (70), showed a gene dosage-dependent reduction in

359 TAU58/Trem $2^{4 /+}$ and TAU58/Trem $2^{4 / 4}$ brains. Conversely, the homeostatic marker Siglech was increased in

360 TAU58/Trem $2^{4 /+}$ and TAU58/Trem $2^{4 / \Delta}$ brains compared to TAU58/Trem $2^{+/+}$mice. Complement factor C4,

361 which was significantly reduced in TAU58/Trem $2^{+/+}$brains compared to Trem $2^{+/+}$controls, reverted to control-

362 like levels in TAU58/Trem $2^{4 /+}$ and TAU58/Trem $2^{4 / 4}$ mice. ApoE, a marker of disease-associated microglia

363 (DAM), which was increased in TAU58/Trem $2^{+/+}$mice, was reduced to control-like levels in TAU58/Trem $2^{4 / 4}$

364 brains. Taken together, Trem 2 depletion in TAU58 mice (i.e. TAU58/Trem $2^{4 / \Delta}$ ) retained microglia in the resting

365 state, while reduction of Trem2 (i.e. TAU58/Trem $2^{4 /+}$ ) showed intermediate microglial gene activation

366 compared to TAU58/Trem2 $2^{+/+}$littermates.

Trem 2 reduction results in increased tau spreading. Microglia have been previously shown to contribute to propagation of tau pathology (14-16). To understand the contribution of tau spreading towards the augmentation of behavioural and neuropathology in TAU58/Trem $2^{4 / 4}$, we used a previously established adeno-associated virus (AAV) model of in vivo tau propagation, AAV1-eGFP-2a-P301L-tau $(55,56)$. Briefly, neurons express eGFP and P301L tau as individual proteins after co-translational cleavage of the peptide chain induced by a viral 2a peptide sequence (donor neurons; $\mathrm{GFP}^{+} / \mathrm{tau}^{+}$). When tau spreading occurs, donor neurons release $\mathrm{P} 301 \mathrm{~L}$ tau that is then taken up by recipient neurons $\left(\mathrm{GFP}^{-} / \mathrm{tau}^{+}\right)$, whereas donor neurons retain eGFP (Figure 4A). Trem2 $2^{4 / 4}$ and Trem $2^{+/+}$mice were injected with AAV1-eGFP-2a-P301L-tau at 4 weeks of age and analysed 8 weeks post injection. The number of recipient neurons positive for human tau and negative for GFP $\left(\mathrm{GFP}^{-} / \mathrm{tau}^{+}\right)$was determined in horizontal brain sections throughout the ipsilateral and contralateral hippocampus, entorhinal cortex and adjacent cortical areas, at 4 different positions along the dorsal-ventral axis of the brain. We found a significant increase in the overall number of $\mathrm{GFP}^{-} / \mathrm{tau}^{+}$cells averaged across all dorsal-ventral positions for ipsilateral, contralateral and the total brain counts in Trem $2^{4 / \Delta}$ mice compared to Trem $2^{+/+}$controls (Figure 4B and 4C, individual dorsal-ventral positions in Figure S4). The number and distribution heatmaps of GFP-/tau ${ }^{+}$ neurons illustrate the increased and more widespread spreading of tau in Trem $2^{\Delta / \Delta}$ mice than Trem $2^{+/+}$controls 
(Figure 4D). Taken together, the depletion of Trem 2 appears to enhance tau pathology due to increased tau spreading in the brain.

\section{Discussion}

387

In the present study, we show that reducing Trem 2 accelerates phenotypes of P301S tau transgenic TAU58 mice in early disease stages. These deficits were accompanied by increased levels of tau phosphorylated at Serine 422, a late stage pathology marker (71), in young TAU58/Trem $2^{4 / 4}$ mice, as well as by reduced microglial activation compared to TAU58 mice. Using an independent in vivo model for tau spreading, we showed that loss of Trem 2 leads to an increased inter-neuronal propagation of pathological tau.

TREM2 limits tau pathology and associated functional deficits. Our study supports the emerging consensus that reduced TREM2 function/levels are associated with increased tau pathology in transgenic mice $(46,49,50)$. Experimental differences (e.g. mouse lines, genetic backgrounds and ages used) may explain the deviating findings of heterozygous Trem2-deficient mice augmenting tau phosphorylation in tau transgenic mice in one study, although even in these mice brains were protected from atrophy (48). Recent studies have connected the role of TREM 2 in tau pathology to $A \beta$, reporting increased tau pathology in the vicinity and/or presence of $A \beta$ pathology (72,73). Mechanistically, increased tau phosphorylation upon Trem 2 reduction/depletion in tau transgenic models has been linked to increased kinase activity (46). Reports of functional changes induced by Trem 2 reduction were limited to studies using virus-mediated localized knockdown of Trem 2 that exacerbated spatial learning deficits of aged P301S tau transgenic mice (49), while overexpression of TREM2 rescued these deficits (38). Here, we show that Trem 2 reduction accelerated onset of behavioural and motor deficits of TAU58 mice in a gene dosage-dependent manner in early disease stages which tapered out in aged animals.

TREM2 confers disease-related microglial profiles in tau pathology. TREM2 has been implicated in the activation of DAM in mouse models of $\mathrm{AD}$ and human disease associated with $\mathrm{A} \beta$ pathology (74). DAM in $\mathrm{AD}$ and other disease mouse models have been identified by single-cell mRNA profiling, revealing activationassociated gene signatures distinct from resting/homeostatic microglia $(74,75)$. Here we used CyTOF to profile isolated microglia based on differential surface marker expression $(64,66)$ as a novel and alternative approach to determine cell signatures associated with tau pathology in mice. Since this method has not been applied in tau transgenic mice (or any other $\mathrm{AD}$ mouse model), direct comparison to previous reports and mRNA signatures is difficult. However, the DAM marker CD11c (=Itgax), that is upregulated in mRNA signature profiling of A $\beta$ forming AD mouse brains (73-75), identified a distinct TREM2-dependent population that is increased in 
413 TAU58 mice. Conversely, P2ry12, which distinguishes resting/homeostatic microglia from other populations

414 (and in particular from DAM) in mRNA signature profiles in tau and APP transgenic mice (73), also

415 distinguished resting/homeostatic from activated/DAM microglia by CyTOF in TAU58 mice. P2ry12 and

416 Cx3cr1 are part of the microglial homeostatic mRNA signature reported to be increased in Trem $2^{-/}$mice and

417 increased in aging $\mathrm{A} \beta$-forming $\mathrm{AD}$ mice (74-76) and are lead markers of resting/homeostatic microglia by

418 CyTOF in our experiments in TAU58 and Trem $2^{4 / \Delta}$ mice. While DAM in TAU58/Trem $2^{4 / \Delta}$ brains were reduced

419 to the level of littermate controls, DAMs remained high in TAU58/Trem $2^{4 /+}$ brains. In contrast, mRNA

420 quantification showed that microglial marker gene expression in TAU58/Trem $2^{4 /+}$ brains aligned with

421 TAU58/Trem $2^{4 / \Delta}$ brains, rather than that of TAU58 mice. This suggests that DAM in TAU58/Trem $2^{4 /+}$ brains

422 may have adopted an intermediate activation state that is not captured by the current microglial CyTOF panel.

423 Nevertheless, we identified Trem2-dependent DAM in TAU58 mice using CyTOF.

TREM2 limits the spreading of tau pathology. Recent evidence suggested that TREM2 limits tau pathology progression in an $A \beta$-dependent context in tau transgenic $p R 5$ mice (73). In fact, $A \beta$ has been shown to enhance tau pathology progression also in other models $(77,78)$. This may explain why A $\beta$ was necessary for Lee et al. (73) to detect tau pathology progression in pR5 mice, which have a low and localized expression of transgenic tau, and show per se only slow progression of tau pathology, as shown by us previously (71). Here, using a previously established model for tracing locally expressed human P301L mutant tau in mouse brains $(55,56)$, we show for the first time that TREM2 limits tau protein spreading, notably, independent of A $\beta$. AAV-mediated human tau expression is expected to be comparable or higher than transgenic human tau expression in TAU58 mice $(51,55,56)$. This suggests that a certain threshold concentration of tau in donor neurons may be required for tau spreading between neurons. During cell-to-cell spreading, tau is released by donor neurons and then takenup by recipient neurons. Here, increased spreading of tau upon reduction of Trem 2 indicated that Trem 2 itself, or the associated microglia activation state (DAM: TAU58/Trem $2^{+/+}>$TAU58/Trem $2^{4 / 4}$ ) may be needed for efficient clearance of released tau from the extracellular space. Alternatively, the uptake of tau by recipient neurons - and hence spreading - may be enhanced in Trem $2^{4 / \Delta}$ mice, for example through a mechanism involving DAM activation as an uptake inhibitor signal for neurons. Interestingly and in line with our findings, tau spreading was increased by the depletion of the TREM2 downstream effector TYROBP/DAP12 in mice 


\section{Conclusion}

443 In summary, our data support a disease-limiting function of TREM2 on tau pathology due to directly limiting

444 neuron-to-neuron transmission of tau proteins, decreasing pathological tau phosphorylation, and, therefore,

445 slowing disease progression. These findings support the role of microglia in the propagation of tau pathology

446 (15). Together with TREM2 polymorphisms increasing the risk of frontotemporal dementia with tau pathology

447 (80), our study provides important tau focused support for the potential therapeutic benefit of agonistic TREM2

448 therapies $(81,82)$ in $\mathrm{AD}$ and tau-driven neurodegenerative diseases. 
$451 \mathrm{~A} \beta$, Amyloid- $\beta$; AD, Alzheimer's disease; TREM2, triggering receptor expressed on myeloid cells 2; NFT,

452 neurofibrillary tangles; FTD, frontotemporal dementia; LOAD, late onset Alzheimer's disease; ALS,

453 amyotrophic lateral sclerosis; AAV, adeno-associated virus; sgRNAs, single-guide RNAs; KO, knock out; OF,

454 Open Field test; MWM, Morris Water Maze; EPM, Elevated Plus Maze; BB, Blocking buffer; CyTOF,

455 cytometry by time of flight.

456

457

Declarations

458

Ethics approval and consent to participate

459

All animal experiments were approved by the Animal Ethics Committees of Macquarie University.l

460

461

Consent for publication

462

Not applicable.

463

464 Availability of data and materials

465 All data generated and analysed during this study are included in this published article and its supplementary

466 files. The detailed datasets generated during the current study are available from the corresponding author upon

467 reasonable request.

468

469 Competing interests

470 The authors declare that they have no competing interests related to this study.

471

$472 \quad$ Funding 
473 This study was funded by the National Health \& Medical Research Council (1132524, 1143848, 1123564,

474 1136241), the Australian Research Council (DP 210101957) and Macquarie University to YDK or LMI. The

475 funding bodies were not involved in the design of the study or the collection, analysis, and interpretation of the

476 data nor in writing the manuscript.

477

\section{Author's contributions}

479 AFF, CK, YDK and LMI designed experiments. AFF, CA, AT, SG, CK, AvH, JvdH, YD, MP, TB, FB and FD

480 performed experiments. SW and BTH provided essential reagents. CK, YDK and LMI supervised the study.

481 YDK and LMI have obtained funding. AFF, YDK and LMI wrote the first manuscript draft. All authors edited

482 the manuscript.

483

\section{Acknowledgments}

485 The authors thank Yijun Lin for the production of the AAV and the Central Animal Facility (CAF) staff for 486 animal care

487 
1. Batsch NL, Mittelman MS. World Alzheimer Report 2012 [Internet]. Alzheimer's Disease International. 2012. 1-80 p. Available from: papers2://publication/uuid/889ADA69-3B6E-4DCC-BF40936650E90072

492

2. Ferri CP, Prince M, Brayne C. Alzheimer's Disease International. Global prevalence of dementia: A Delphi consensus study. Lancet. 2005;366(9503):2112-7.

3. Prince M, Jackson J. World Alzheimer Report 2009. Alzheimer’s Disease International. 2009.

4. prince martin. World Alzheimer Report. 2015; Available from: https://www.alz.co.uk/research/WorldAlzheimerReport2015.pdf

5. Ittner LM, Götz J. Amyloid- $\beta$ and tau - A toxic pas de deux in Alzheimer's disease. Nat Rev Neurosci. 2011;

6. Rosso SM, Kaat LD, Baks T, Joosse M, De Koning I, Pijnenburg Y, et al. Frontotemporal dementia in The Netherlands: Patient characteristics and prevalence estimates from a population-based study. Brain.

7. Stevens M, Van Duijn CM, Kamphorst W, De Knijff P, Heutink P, Van Gool WA, et al. Familial aggregation in frontotemporal dementia. Neurology. 1998;

8. Ferrari R, Hernandez DG, Nalls MA, Rohrer JD, Ramasamy A, Kwok JBJ, et al. Frontotemporal dementia and its subtypes: A genome-wide association study. Lancet Neurol. 2014;

9. Götz J, Deters N, Doldissen A, Bokhari L, Ke Y, Wiesner A, et al. A decade of tau transgenic animal models and beyond. In: Brain Pathology. 2007.

10. Braak H, Braak E. Neuropathological stageing of Alzheimer-related changes. Acta Neuropathologica. 1991.

11. De Calignon A, Polydoro M, Suárez-Calvet M, William C, Adamowicz DH, Kopeikina KJ, et al. Propagation of Tau Pathology in a Model of Early Alzheimer's Disease. Neuron. 2012;

512 12. Liu L, Drouet V, Wu JW, Witter MP, Small SA, Clelland C, et al. Trans-synaptic spread of tau pathology in vivo. PLoS One. 2012; 
13. Frost B, Jacks RL, Diamond MI. Propagation of Tau misfolding from the outside to the inside of a cell. J Biol Chem. 2009;284(19):12845-52.

14. Maphis N, Xu G, Kokiko-Cochran ON, Jiang S, Cardona A, Ransohoff RM, et al. Reactive microglia drive tau pathology and contribute to the spreading of pathological tau in the brain. Brain. 2015;

15. Asai H, Ikezu S, Tsunoda S, Medalla M, Luebke J, Haydar T, et al. Depletion of microglia and inhibition of exosome synthesis halt tau propagation. Nat Neurosci. 2015;

16. Hopp SC, Lin Y, Oakley D, Roe AD, Devos SL, Hanlon D, et al. The role of microglia in processing and spreading of bioactive tau seeds in Alzheimer's disease 11 Medical and Health Sciences 1109 Neurosciences. J Neuroinflammation. 2018; Internalization of Tau by Microglia in Vitro and in Vivo. J Alzheimer's Dis. 2016; pathological tau is enhanced by an anti-tau monoclonal antibody. Sci Rep. 2015;

19. Hansen D V., Hanson JE, Sheng M. Microglia in Alzheimer's disease. Journal of Cell Biology. 2018.

20. Takatori S, Wang W, Iguchi A, Tomita T. Genetic Risk Factors for Alzheimer Disease: Emerging Roles of Microglia in Disease Pathomechanisms. In: Advances in Experimental Medicine and Biology. 2019.

21. Guerreiro R, Wojtas A, Bras J, Carrasquillo M, Rogaeva E, Majounie E, et al. TREM2 variants in Alzheimer's disease. N Engl J Med. 2013; associated with the risk of Alzheimer's disease. N Engl J Med. 2013; and Chorea Associated with a Compound Heterozygous TREM2 Mutation. J Alzheimers Dis. 2018;

24. Williamson JC, Larner AJ. Behavioral variant frontotemporal dementia-like syndrome with novel as a risk factor for sporadic amyotrophic lateral sclerosis. JAMA Neurol. 2014; 
26. E. K, K. OG. TREM2 genetic deletion exacerbates the disease course in the SOD1G93A mouse model of ALS. Neurodegener Dis. 2017;

27. Rayaprolu S, Mullen B, Baker M, Lynch T, Finger E, Seeley WW, et al. TREM2 in neurodegeneration: Evidence for association of the p.R47H variant with frontotemporal dementia and Parkinson's disease. Mol Neurodegener. 2013;

35. Atagi Y, Liu CC, Painter MM, Chen XF, Verbeeck C, Zheng H, et al. Apolipoprotein E is a ligand for triggering receptor expressed on myeloid cells 2 (TREM2). J Biol Chem. 2015; 
38. Jiang T, Tan L, Zhu XC, Zhang QQ, Cao L, Tan MS, et al. Upregulation of TREM2 ameliorates neuropathology and rescues spatial cognitive impairment in a transgenic mouse model of Alzheimer's disease. Neuropsychopharmacology. 2014;

39. Wang Y, Cella M, Mallinson K, Ulrich JD, Young KL, Robinette ML, et al. TREM2 lipid sensing sustains the microglial response in an Alzheimer's disease model. Cell. 2015;

40. Jay TR, Miller CM, Cheng PJ, Graham LC, Bemiller S, Broihier ML, et al. TREM2 deficiency eliminates TREM2+ inflammatory macrophages and ameliorates pathology in Alzheimer's disease mouse models. J Exp Med. 2015;

41. Ulrich JD, Finn MB, Wang Y, Shen A, Mahan TE, Jiang H, et al. Altered microglial response to A $\beta$ plaques in APPPS1-21 mice heterozygous for TREM2. Mol Neurodegener. 2014;

42. Ulrich JD, Holtzman DM. TREM2 Function in Alzheimer's Disease and Neurodegeneration. ACS Chemical Neuroscience. 2016.

43. Yeh FL, Hansen D V., Sheng M. TREM2, Microglia, and Neurodegenerative Diseases. Trends in Molecular Medicine. 2017.

44. Lee CYD, Daggett A, Gu X, Jiang LL, Langfelder P, Li X, et al. Elevated TREM2 Gene Dosage Reprograms Microglia Responsivity and Ameliorates Pathological Phenotypes in Alzheimer's Disease Models. Neuron. 2018;

45. Jay TR, Hirsch AM, Broihier ML, Miller CM, Neilson LE, Ransohoff RM, et al. Disease progressiondependent effects of TREM2 deficiency in a mouse model of Alzheimer's disease. J Neurosci. 2017;

46. Bemiller SM, McCray TJ, Allan K, Formica S V., Xu G, Wilson G, et al. TREM2 deficiency exacerbates tau pathology through dysregulated kinase signaling in a mouse model of tauopathy. Mol Neurodegener. 2017;

47. Leyns CEG, Ulrich JD, Finn MB, Stewart FR, Koscal LJ, Serrano JR, et al. TREM2 deficiency attenuates neuroinflammation and protects against neurodegeneration in a mouse model of tauopathy. Proc Natl Acad Sci U S A. 2017;

48. Sayed FA, Telpoukhovskaia M, Kodama L, Li Y, Zhou Y, Le D, et al. Differential effects of partial and complete loss of TREM2 on microglial injury response and tauopathy. Proc Natl Acad Sci U S A. 2018; 
49. Jiang T, Tan L, Zhu XC, Zhou JS, Cao L, Tan MS, et al. Silencing of TREM2 exacerbates tau pathology, neurodegenerative changes, and spatial learning deficits in P301S tau transgenic mice. Neurobiol Aging. 2015;

50. Jiang T, Zhang YD, Chen Q, Gao Q, Zhu XC, Zhou JS, et al. TREM2 modifies microglial phenotype and provides neuroprotection in P301S tau transgenic mice. Neuropharmacology. 2016;

51. van Eersel J, Stevens CH, Przybyla M, Gladbach A, Stefanoska K, Chan CKX, et al. Early-onset axonal pathology in a novel P301S-Tau transgenic mouse model of frontotemporal lobar degeneration. Neuropathol Appl Neurobiol. 2015;

52. Przybyla M, Stevens CH, van der Hoven J, Harasta A, Bi M, Ittner A, et al. Disinhibition-like behavior in a P301S mutant tau transgenic mouse model of frontotemporal dementia. Neurosci Lett. 2016;

53. Przybyla M, van Eersel J, van Hummel A, van der Hoven J, Sabale M, Harasta A, et al. Onset of hippocampal network aberration and memory deficits in P301S tau mice are associated with an early gene signature. Brain. 2020;

54. van der Hoven J, van Hummel A, Przybyla M, Asih PR, Gajwani M, Feiten AF, et al. Contribution of endogenous antibodies to learning deficits and astrocytosis in human P301S mutant tau transgenic mice. Sci Rep. 2020;

55. Wegmann S, Maury EA, Kirk MJ, Saqran L, Roe A, DeVos SL, et al. Removing endogenous tau does not prevent tau propagation yet reduces its neurotoxicity. EMBO J. 2015;34(24):3028-41.

56. Wegmann S, Bennett RE, Delorme L, Robbins AB, Hu M, McKenzie D, et al. Experimental evidence for the age dependence of tau protein spread in the brain. Sci Adv. 2019;

57. Delerue F, Ittner LM. Generation of genetically modified mice through the microinjection of oocytes. J Vis Exp. 2017;

58. Van Hummel A, Bi M, Ippati S, Van Der Hoven J, Volkerling A, Lee WS, et al. No overt deficits in aged tau-deficient C57B1/6.Mapttm1(EGFP)kit GFP knockin mice. PLoS One. 2016;

59. Ke YD, van Hummel A, Stevens CH, Gladbach A, Ippati S, Bi M, et al. Short-term suppression of A315T mutant human TDP-43 expression improves functional deficits in a novel inducible transgenic mouse model of FTLD-TDP and ALS. Acta Neuropathol. 2015; 

excitotoxic brain damage in an animal model of stroke. Nat Commun. 2017;8(1).

61. Van Eersel J, Bi M, Ke YD, Hodges JR, Xuereb JH, Gregory GC, et al. Phosphorylation of soluble tau differs in Pick's disease and Alzheimer's disease brains. J Neural Transm. 2009;

62. Mei HE, Leipold MD, Schulz AR, Chester C, Maecker HT. Barcoding of Live Human Peripheral Blood Mononuclear Cells for Multiplexed Mass Cytometry. J Immunol. 2015;194(4).

63. Hartmann FJ, Bernard-Valnet R, Quériault C, Mrdjen D, Weber LM, Galli E, et al. High-dimensional single-cell analysis reveals the immune signature of narcolepsy. J Exp Med. 2016;213(12).

64. Krieg C, Nowicka M, Guglietta S, Schindler S, Hartmann FJ, Weber LM, et al. Author Correction: High-dimensional single-cell analysis predicts response to anti-PD-1 immunotherapy. Nat Med. cytometry data with bead standards. Cytom Part A. 2013;83 A(5).

66. Robinson MD, Nowicka M, Krieg C, Weber LM, Hartmann FJ, Guglietta S, et al. CyTOF workflow: Differential discovery in high-throughput high-dimensional cytometry datasets. F1000Research. 2017;6.

67. Watt G, Przybyla M, Zak V, van Eersel J, Ittner A, Ittner LM, et al. Novel Behavioural Characteristics of Male Human P301S Mutant Tau Transgenic Mice - A Model for Tauopathy. Neuroscience. 2020;

68. Götz J, Chen F, Van Dorpe J, Nitsch RM. Formation of neurofibrillary tangles in P301L tau transgenic mice induced by A $\beta 42$ fibrils. Science (80- ). 2001;293(5534).

69. Kierdorf K, Prinz M. Factors regulating microglia activation. Frontiers in Cellular Neuroscience. 2013.

70. Hoshi T, Toyama T, Shinozaki Y, Koizumi S, Lee JY, Naganuma A, et al. Evaluation of M1-microglial activation by neurotoxic metals using optimized organotypic cerebral slice cultures. J Toxicol Sci. 2019;44(7).

71. Deters N, Ittner LM, Götz J. Divergent phosphorylation pattern of tau in P301L tau transgenic mice. Eur J Neurosci. 2008; 
73. Lee SH, Meilandt WJ, Xie L, Gandham VD, Ngu H, Barck KH, et al. Trem2 restrains the enhancement of tau accumulation and neurodegeneration by $\beta$-amyloid pathology. Neuron. 2021;109(8).

650

651

652

653

74. Zhou Y, Song WM, Andhey PS, Swain A, Levy T, Miller KR, et al. Human and mouse single-nucleus transcriptomics reveal TREM2-dependent and TREM2-independent cellular responses in Alzheimer's disease. Nat Med. 2020;26(1).

75. S. K, C. M, R. C, C. B, N. C, R. EF, et al. The TREM2-APOE Pathway Drives the Transcriptional Phenotype of Dysfunctional Microglia in Neurodegenerative Diseases. Vol. 47, Immunity. 2017.

76. Götzl JK, Brendel M, Werner G, Parhizkar S, Sebastian Monasor L, Kleinberger G, et al. Opposite microglial activation stages upon loss of PGRN or TREM 2 result in reduced cerebral glucose metabolism . EMBO Mol Med. 2019;11(6).

77. Bennett RE, DeVos SL, Dujardin S, Corjuc B, Gor R, Gonzalez J, et al. Enhanced Tau Aggregation in the Presence of Amyloid $\beta$. Am J Pathol. 2017;187(7).

78. Pooler AM, Polydoro M, Maury EA, Nicholls SB, Reddy SM, Wegmann S, et al. Amyloid accelerates tau propagation and toxicity in a model of early Alzheimer's disease. Acta Neuropathol Commun. $2015 ; 3$.

79. Audrain M, Haure-Mirande JV, Wang M, Kim SH, Fanutza T, Chakrabarty P, et al. Integrative approach to sporadic Alzheimer's disease: deficiency of TYROBP in a tauopathy mouse model reduces C1q and normalizes clinical phenotype while increasing spread and state of phosphorylation of tau. Mol Psychiatry. 2019;

80. A. L-C, A. L, C. T-M, E. G-C, J.C. G-V, P. A-R, et al. TREM2 variant p.His157Tyr in Colombian patients with frontotemporal dementia. J Neurochem. 2016;138.

81. Lewcock JW, Schlepckow K, Di Paolo G, Tahirovic S, Monroe KM, Haass C. Emerging Microglia Biology Defines Novel Therapeutic Approaches for Alzheimer’s Disease. Vol. 108, Neuron. 2020.

82. Price BR, Sudduth TL, Weekman EM, Johnson S, Hawthorne D, Woolums A, et al. Therapeutic Trem2 activation ameliorates amyloid-beta deposition and improves cognition in the 5XFAD model of amyloid deposition. J Neuroinflammation. 2020;17(1). 
675 Figure $1 \mid$ Reducing Trem2 accelerated functional deficits in P301S tau transgenic TAU58 mice.

676 (A) Significant reduction and loss of Trem 2 mRNA expression in brains of respective $\operatorname{Trem} 2^{4 /+}$ and $\operatorname{Trem} 2^{4 / \Delta}$

677 mice as compared to Trem $2^{+/+}$littermates ( $=4 ; * * *, p<0.001 ; * * * *, p<0.0001$ [ANOVA, Student's t-test]).

678 Schematic of breeding strategy to obtain littermates with different genotypes for this study (colour coding of 679 genotypes apply to all figures and panels. (C) Latency to find the hidden platform during consecutive daily 680 Morris water maze (MWM) acquisition trials (left) and linear regression slopes (middle) in TAU58/Trem $2^{4 / 4}$ 681 mice compared to all other genotypes ( $\mathrm{n}=9-12$; $^{*}, \mathrm{p}<0.05$ [ANOVA, Student's $t$-test]). Comparable times spent in the target quadrant $(\mathrm{Q} 1)$ as opposed to other quadrants (Q2-Q4) during MWM probe trials for all genotypes tested. (D) Latency to fall off an accelerating rotating rod for TAU58/Trem $2^{+/+}$, TAU58/Trem $2^{4 /+}$ and TAU58/Trem $2^{4 / \Delta}$ mice and Trem $2^{+/+}, \operatorname{Trem} 2^{4 /+}$ and $\operatorname{Trem} 2^{4 / \Delta}$ littermate controls at indicated ages $(\mathrm{n}=10-29 ; *$, $\mathrm{p}<0.05 ; * *, \mathrm{p}<0.01 ; * * *, \mathrm{p}<0.001 ; * * * *, \mathrm{p}<0.0001$ [ANOVA, Student's $t$-test] (E) Time to cross a narrow beam and number of foot slips during the crossing for TAU58/Trem $2^{+/+}$, TAU58/Trem $2^{4 /+}$ and TAU58/Trem $2^{4 / \Delta}$ mice and Trem $2^{+/+}, \operatorname{Trem} 2^{4 /+}$ and Trem $2^{4 / 4}$ littermate controls at indicated ages ( 1 and 2 months top row, 3 and 6 months bottom row) ( 1 and 2 months $\mathrm{n}=10-14,3$ and 6 months $\mathrm{n}=3-19 ;{ }^{*}, \mathrm{p}<0.05 ; * *, \mathrm{p}<0.01 ; * * *, \mathrm{p}<0.001$; during one or both test trials at 3 and 6 months of age comparing TAU58/Trem $2^{+/+}$, TAU58/Trem $2^{4 /+}$ and TAU58/Trem $2^{4 / \Delta}$ mice. (G) Time spent in the open arms of the elevated plus maze for TAU58/Trem $2^{+/+}$, TAU58/Trem $2^{4 /+}$ and TAU58/Trem $2^{4 / \Delta}$ mice and Trem $2^{+/+}, \operatorname{Trem} 2^{4 /+}$ and $\operatorname{Trem} 2^{4 / \Delta}$ littermate controls at indicated ages $(1$ and 2 months $\mathrm{n}=9-14,3$ and 6 months $\mathrm{n}=5-20 ; *, \mathrm{p}<0.05 ; * *, \mathrm{p}<0.01 ; * * *, \mathrm{p}<0.001 ; * * * *$, $\mathrm{p}<0.0001$ [ANOVA, Student's $t$-test]).

695

Figure 2 | Augmented late-stage tau phosphorylation upon Trem2 reduction in P301S tau transgenic TAU58 mice.

(A) Representative immunofluorescence staining with an antibody specific for phosphorylated Serine 214 $(\mathrm{pS} 214)$ of tau in TAU58/Trem $2^{+/+}$, TAU58/Trem $2^{4 /+}$ and TAU58/Trem $2^{4 / \Delta}$ brains and Trem $2^{+/+}$, Trem $2^{4 /+}$ and $\operatorname{Trem} 2^{4 / \Delta}$ littermate controls at indicated ages. Nuclei are stained with DAPI (blue). Graphs show stereological quantification of pS214-stained brain sections in these brains at indicated ages $(\mathrm{n}=4-6 ; *, \mathrm{p}<0.05 ; * *, \mathrm{p}<0.01$; $* * *, \mathrm{p}<0.001 ; * * * *, \mathrm{p}<0.0001$ [ANOVA]). (B) Representative immunofluorescence staining with an antibody specific for late-stage phosphorylated Serine $422(\mathrm{pS} 422)$ of tau in TAU58/Trem2 $2^{+/+}$, TAU58/Trem2 $2^{4 /+}$ and 

quantification of pS422-stained brain sections in these brains at indicated ages $\left(\mathrm{n}=4-6 ;{ }^{*}, \mathrm{p}<0.05 ;{ }^{* *}, \mathrm{p}<0.01\right.$; ***, $\mathrm{p}<0.001 ;{ }^{* * * *}, \mathrm{p}<0.0001$ [ANOVA]). No pS422 staining was found in $\operatorname{Trem} 2^{+/+}, \operatorname{Trem} 2^{4 /+}$ and $\operatorname{Trem} 2^{4 / 4}$ littermate controls at all ages. Scale bars, $100 \mu \mathrm{m}$.

Figure 3 | Trem2 reduction diminished microglia activation in P301S tau transgenic TAU58 mice.

(A) Representative immunofluorescence images of hippocampus and motor cortex from TAU58/Trem $2^{+/+}$, TAU58/Trem $2^{1 /+}$ and TAU58/Trem $2^{4 / 4}$ mice co-stained with antibodies to microglial Iba1 (green) and pS396/pS401 tau (PHF-1, red). Arrowheads indicate Iba1-positive cells in the proximity of pS396/pS401stained neurons. Scale bar, $20 \mu \mathrm{m}$. Graphs show quantification of Iba1+ microglia in the proximity of

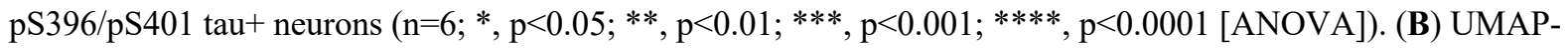
graph of distinct clusters of homeostatic/resting and activated/DAM microglia by CyTOF of single-cell extracts from brains of TAU58/Trem2 $2^{+/+}$, TAU58/Trem $2^{4 /+}$ and TAU58/Trem $2^{4 / \Delta}$ mice and Trem $2^{+/+}$, Trem $2^{4 /+}$ and $\operatorname{Trem} 2^{4 / 4}$ littermate controls. (C) Corresponding heat map of marker expression showed clustering of homeostatic/resting and activated/DAM microglia (D) Comparison of relative abundance of homeostatic/resting and activated/DAM microglia in TAU58/Trem $2^{+/+}$, TAU58/Trem $2^{4 /+}$ and TAU58/Trem $2^{4 / 4}$ mice and Trem $2^{+/+}$, $\operatorname{Trem} 2^{4 /+}$ and Trem $2^{4 / 4}$ littermate controls $\left(\mathrm{n}=3-5 ;{ }^{*}, \mathrm{p}<0.05 ;{ }^{* *}, \mathrm{p}<0.01 ;{ }^{* * *}, \mathrm{p}<0.001 ; * * * *, \mathrm{p}<0.0001\right.$ [ANOVA]). (E) Quantitative real-time PCR of SiglecH, CD16, C4 and ApoE mRNA in hippocampal extracts from TAU58/Trem2 $2^{+/+}$, TAU58/Trem $2^{4 /+}$ and TAU58/Trem $2^{4 / \Delta}$ mice and Trem $2^{+/+}$, Trem $2^{4 /+}$ and Trem $2^{4 / 4}$ littermate controls $\left(\mathrm{n}=2-4 ;{ }^{*}, \mathrm{p}<0.05 ;{ }^{* *}, \mathrm{p}<0.01 ;{ }^{* * *}, \mathrm{p}<0.001 ; * * *, \mathrm{p}<0.0001\right.$ [ANOVA, Student's $t$-test]).

Figure 4 | Trem2 depletion increased spreading of pathological tau.

(A) Schematic of tau spreading mouse model; Unilateral stereotaxic delivery of adeno-associated virus (AAV) for neuronal co-expression of green fluorescence protein (GFP) and human P301L mutant tau into the entorhinal cortex of 4 week-old $\operatorname{Trem} 2^{+/+}$and $\operatorname{Trem} 2^{4 / 4}$ mice. When spreading occurs, $\mathrm{GFP}^{+} / \mathrm{tau}^{+}$donor neurons release tau which is taken up by $\mathrm{GFP}^{-} / \mathrm{tau}^{+}$recipient neurons. (B) Representative immunofluorescent staining for GFP (green) and human tau (red) in ipsilateral and contralateral hippocampi of $\operatorname{Trem}^{+/+}$and $\operatorname{Trem}^{4 / 4}$ mice. Arrowheads indicate recipient neurons $\left(\mathrm{GFP}^{-} / \mathrm{tau}^{+}\right)$in close-up images of the area delineated by broken boxes in hippocampus overview images. Scale bars, $200 \mu \mathrm{m}$ and $20 \mu \mathrm{m}$ respectively. (C) 
for ipsilateral, contralateral and total brain $(\mathrm{n}=9-10 ; *, \mathrm{p}<0.05 ; * *, \mathrm{p}<0.01$; [Student's $t$-test]). (D) Heat maps of tau spreading at distinct levels in the brains of $\operatorname{Trem} 2^{+/+}$and $\operatorname{Trem} 2^{4 / \Delta}$ mice $(\mathrm{n}=9-10)$. The asterisk indicates the injection site.

737

\section{Supplementary Figure Legends}

Figure S1 | Generation of the Trem $2^{4 / 4}$ mouse line.

(A) CRISPR targeting strategy to introduce a deletion $(\Delta)$ into exon 1 of the murine Trem 2 locus, comprising the transcriptional start codon. Arrows indicate position of genotyping primers for identification of mutant alleles. (B) Genome sequencing confirmed a $92 \mathrm{bp}$ deletion in Trem $2^{4 / 4}$ mice. Boxes outline position of guide RNAs used for CRSIPR/Cas9 genome editing projected on the unmodified Trem 2 sequence, highlighting the transcriptional start codon. (C) Example of genotyping PCR showing the mutant band at around 500bp and wild-type band at 600bp in Trem $2^{4 / 4}$ (lane 1), $\operatorname{Trem}^{4 /+}$ (lane 2) and $\operatorname{Trem}^{+/+}$(lane 3) mice.

Figure S2 | Unaffected locomotor activity in TAU58/Trem $2^{1 / 4}$ mice.

(A) Distance travelled during elevated plus maze (EPM) for TAU58/Trem $2^{+/+}$, TAU58/Trem $2^{4 /+}$ and TAU58/Trem $2^{4 / 4}$ mice and Trem $2^{+/+}, \operatorname{Trem} 2^{4 /+}$ and Trem $2^{4 / 4}$ littermate controls. (B) Time in inner zone and distance travelled during open field testing for TAU58/Trem $2^{+/+}$, TAU58/Trem $2^{4 /+}$ and TAU58/Trem $2^{4 / 4}$ mice and $\operatorname{Trem} 2^{+/+}, \operatorname{Trem} 2^{4 /+}$ and $\operatorname{Trem} 2^{4 / 4}$ littermate controls.

752

Figure S3 | Tau phosphorylation and microglia distribution in TAU58/Trem $2^{4 / 4}$ mice

(A) Representative immunofluorescence staining with an antibody specific for phosphorylated Serine 214 (pS214; green) of tau in the cortex of TAU58/Trem $2^{+/+}$, TAU58/Trem $2^{4 /+}$ and TAU58/Trem $2^{4 / 4}$ brains and Trem $2^{+/+}, \operatorname{Trem} 2^{4 /+}$ and $\operatorname{Trem} 2^{4 / 4}$ littermate controls at 2 months of age. Nuclei are stained with DAPI (blue). Graph shows stereological quantification of pS214-stained brain sections in these brains ( $\mathrm{n}=4-6 ;{ }^{* * *}, \mathrm{p}<0.001$; phosphorylated Serine 422 (pS422; green) of tau in the cortex of TAU58/Trem $2^{+/+}$, TAU58/Trem $2^{4 /+}$ and TAU58/Trem $2^{4 / 4}$ brains at 2 months of age. Nuclei are stained with DAPI (blue). Graph shows stereological quantification of pS214-stained brain sections in these brains $(\mathrm{n}=4-6 ; * * *, \mathrm{p}<0.001 ; * * * *, \mathrm{p}<0.0001$ [ANOVA]). (C) Representative immunofluorescence staining with an antibody specific for microglia Iba1 (green) and human tau (red) co-stained for nuclear DAPI (blue) of tau in TAU58/Trem2 $2^{+/+}$, TAU58/Trem $2^{4 /+}$ 
764 and TAU58/Trem $2^{4 / \Delta}$ brains and Trem $2^{+/+}$, Trem $2^{4 /+}$ and Trem $2^{4 / \Delta}$ littermate controls at indicated ages. Graphs

765 show stereological quantification of microglial density in brain sections at indicated ages ( $\mathrm{n}=4-6$; ns, not

766 significant $[\mathrm{ANOVA}])$.

767

768 Figure S4 | Trem2 depletion increased spreading of pathological tau

769 Representative immunofluorescent staining for GFP (green) and human tau (red) in ipsilateral and contralateral

770 hippocampi of Trem $2^{+/+}$and $T r e m 2^{4 / \Delta}$ mice at different depths relative to the Bregma covering the ventral,

771 medial and dorsal hippocampus. Arrowheads indicate recipient neurons $\left(\mathrm{GFP}^{-} / \mathrm{tau}^{+}\right)$in close-up images of the

772 area delineated by broken boxes in hippocampus overview images. Scale bars, $200 \mu \mathrm{m}$ and $20 \mu \mathrm{m}$ respectively. 

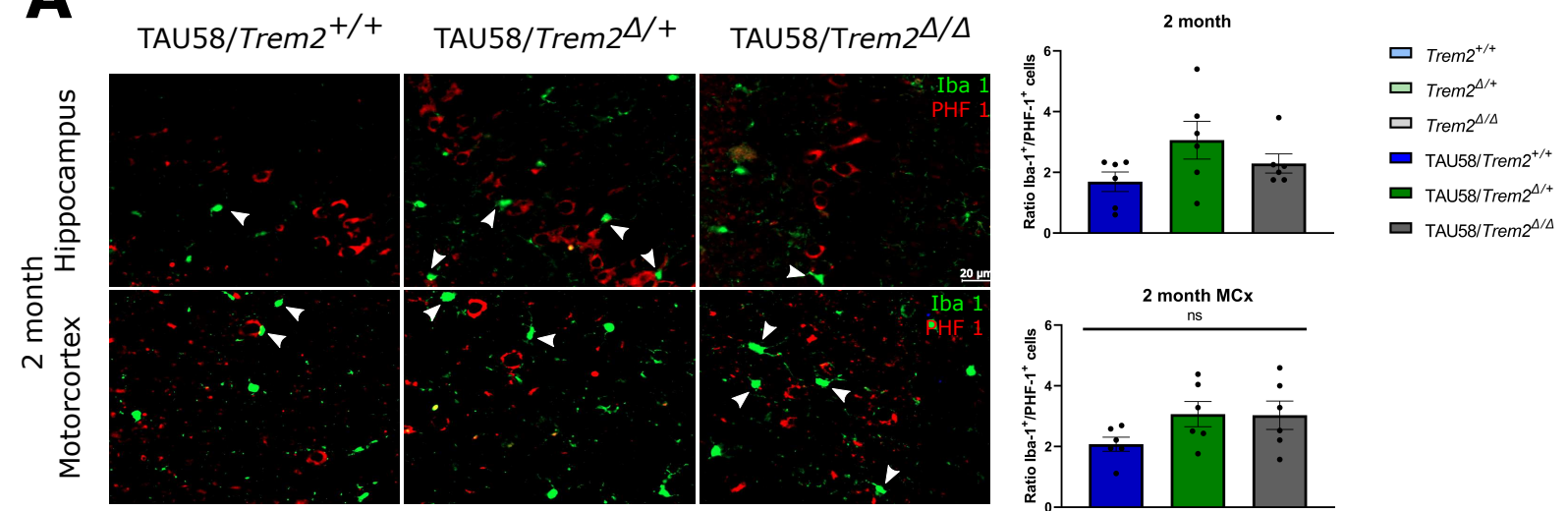

B
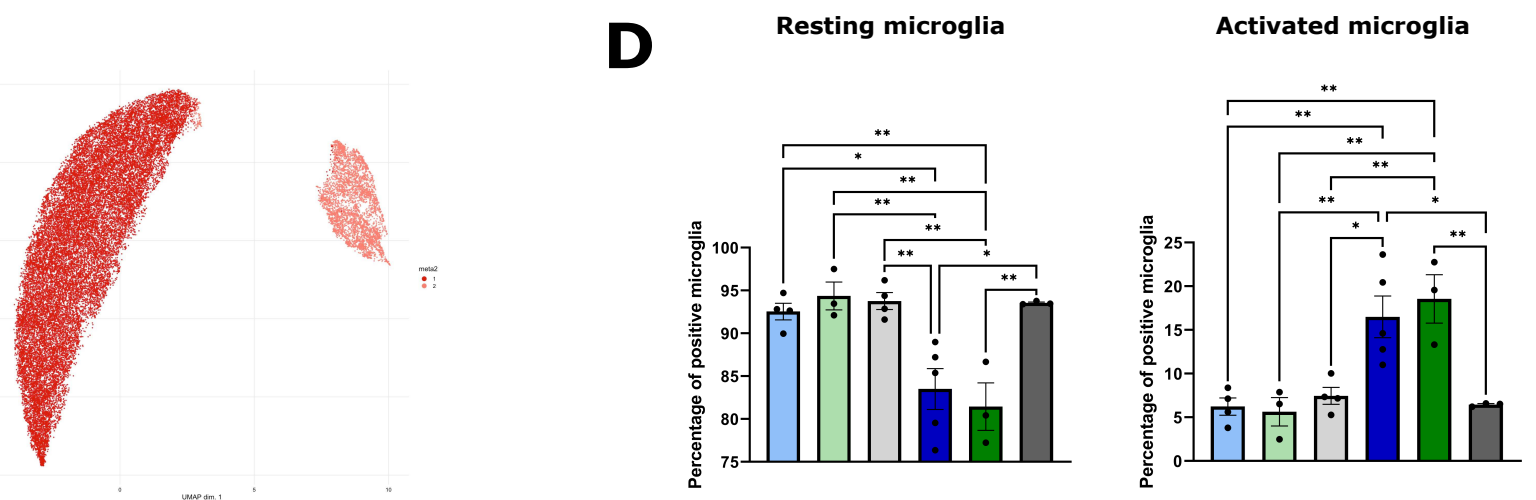

C

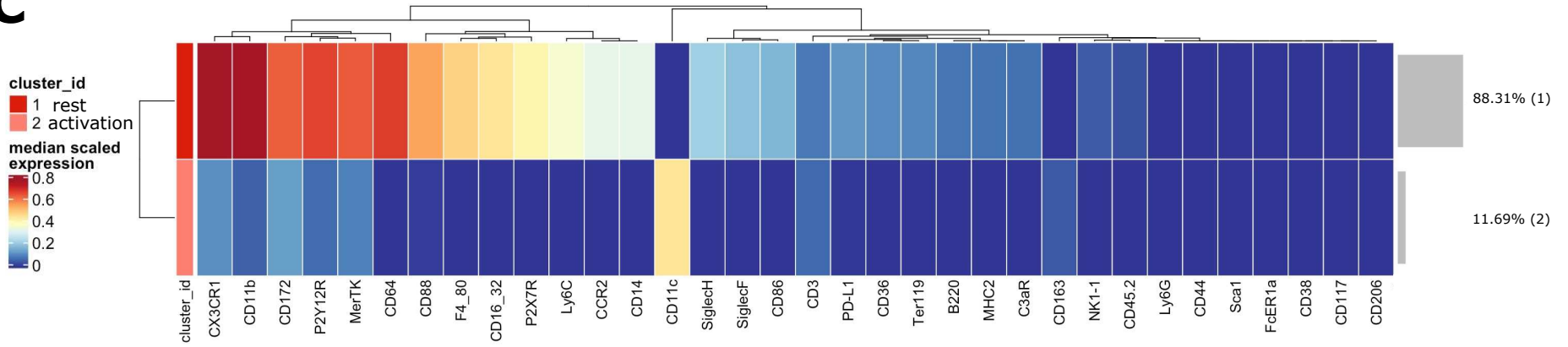

E

Siglech

CD16

C4

ApoE
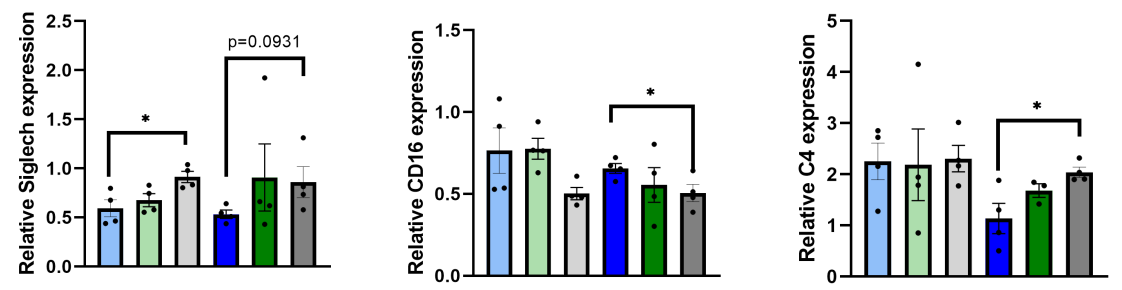

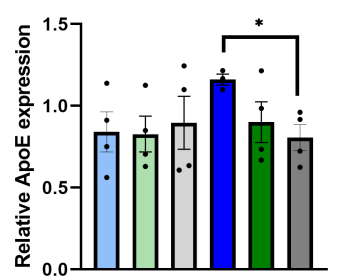


A

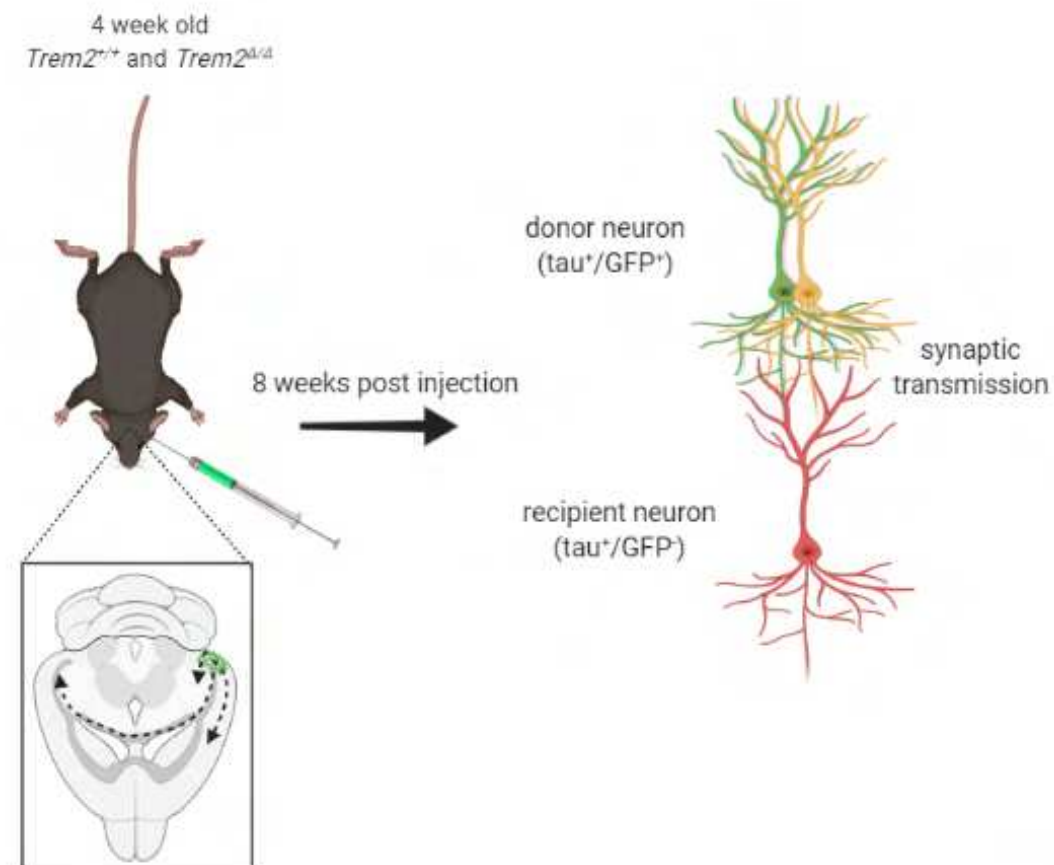

B

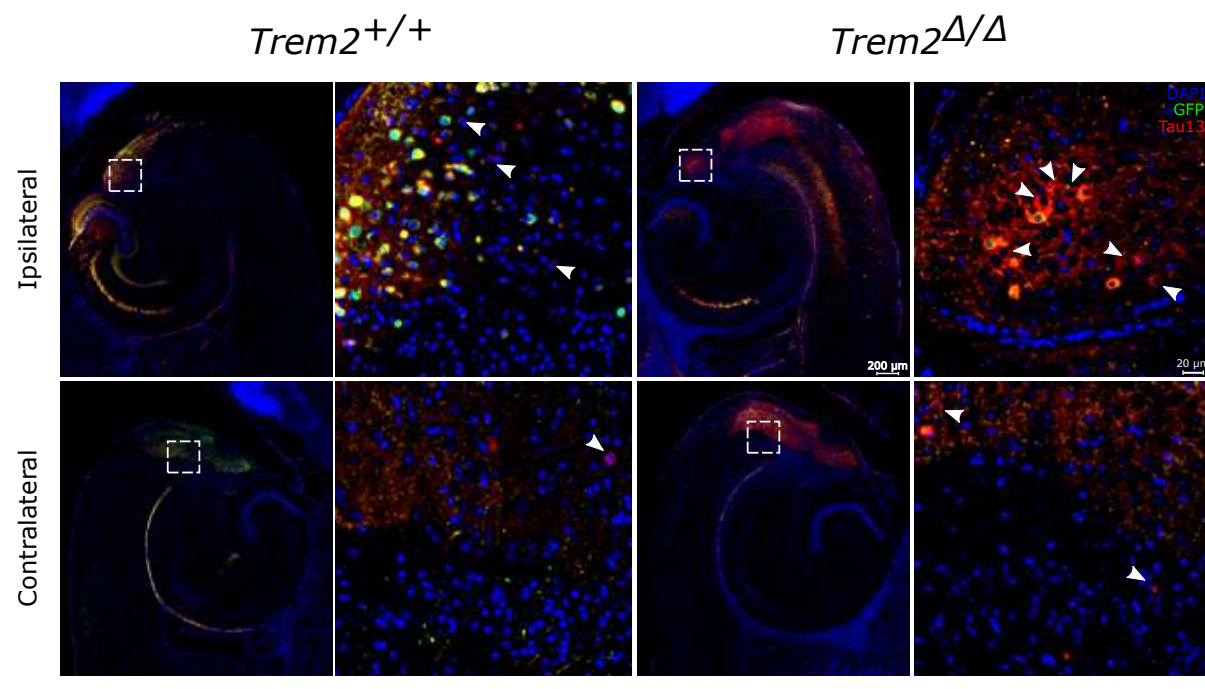

C

Ipsilateral

Contralateral

Total

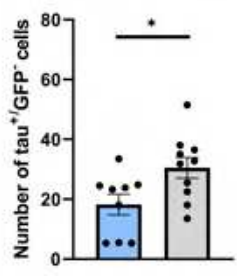

D Trem2 $2^{+/+}$

$\operatorname{Trem}^{\Delta / \Delta}$
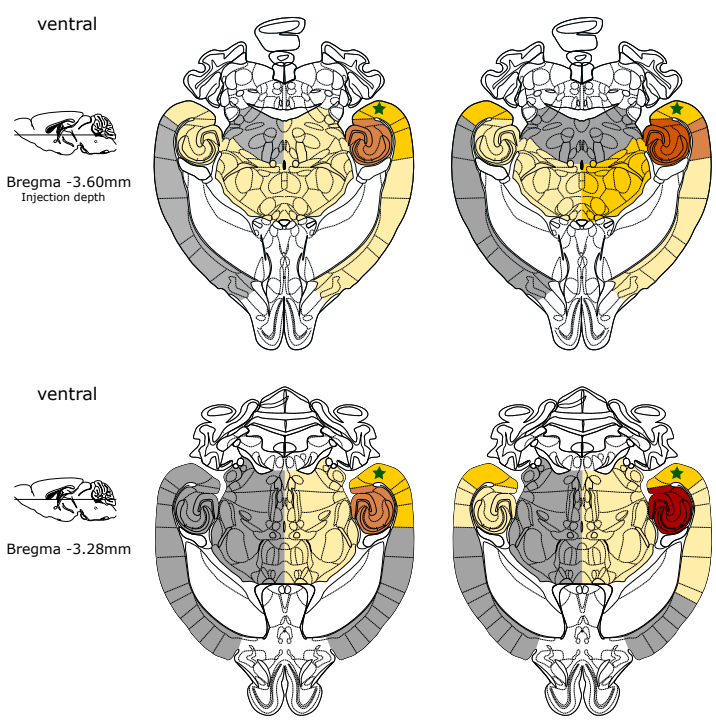

medial

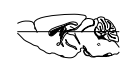

Bregma - $2.68 \mathrm{~mm}$
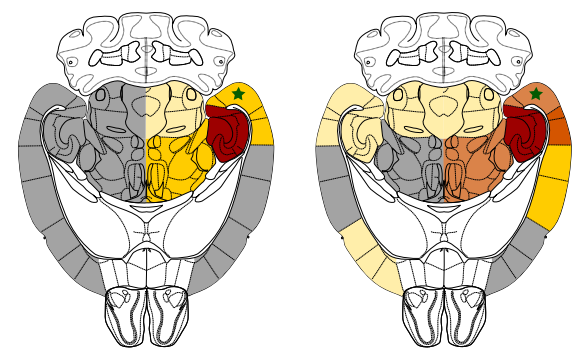

dorsa

Sol
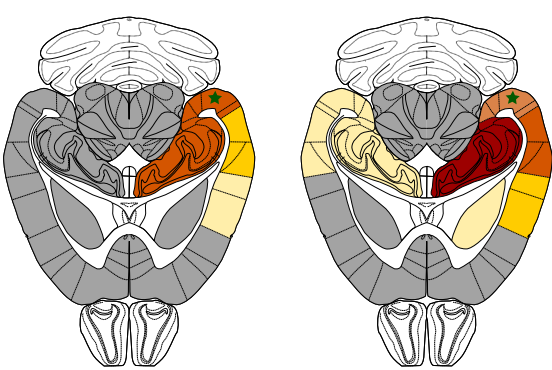

$\star$ injection site

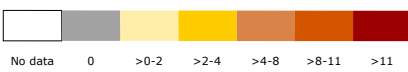

Figure 4 
mouse TREM2

(Chr17)

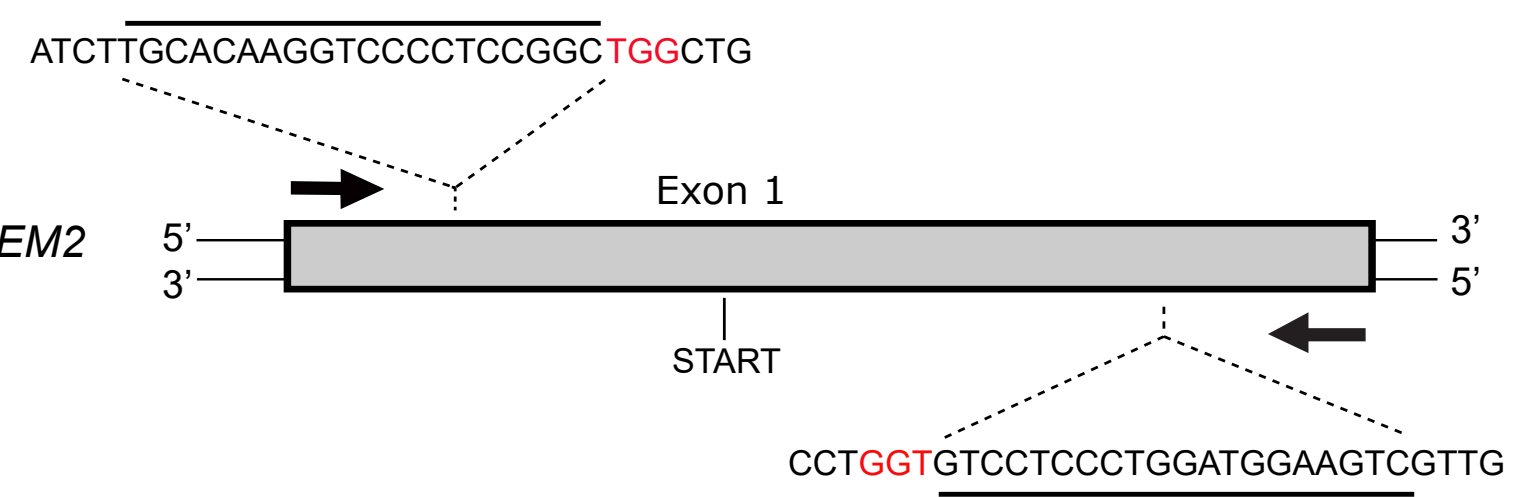

B
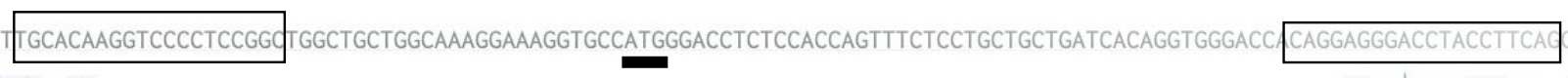

- 92bp

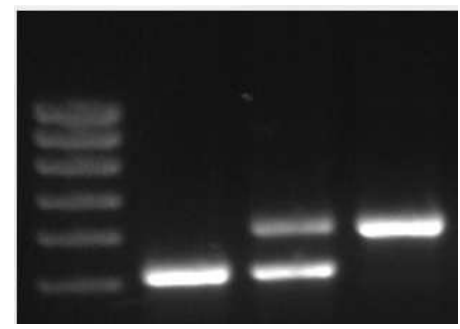

Supplementary Figure 1 

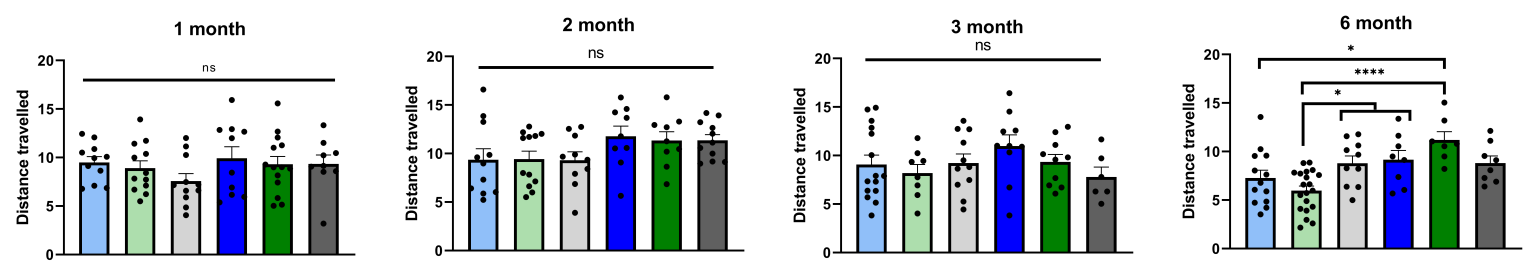

ロ Trem2 $^{+/+}$

ロ $\mathrm{Trem}^{\mathrm{Al+}}$

口 $\operatorname{Trem} 2^{\Delta / \Delta}$

- TAU58/Trem2 ${ }^{+/+}$

- TAU58/Trem $2^{\Delta /+}$ 口 TAU58/Trem2 $2^{\Delta / \Delta}$

B
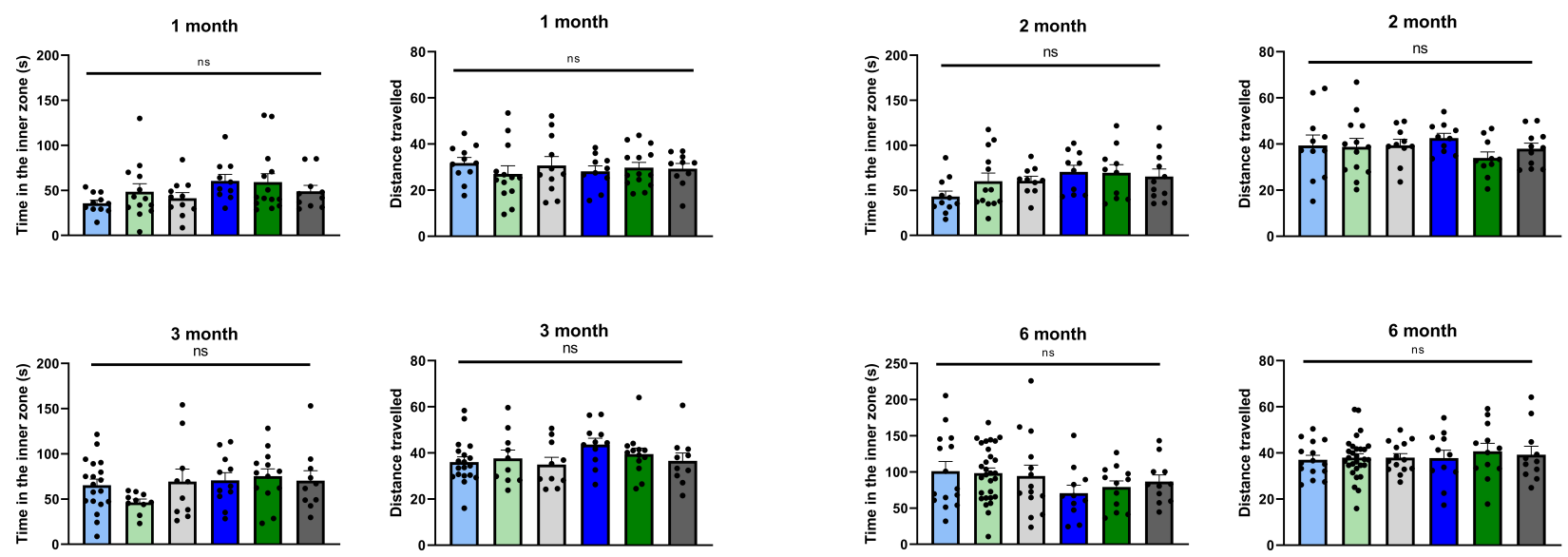

Supplementary Figure 2 


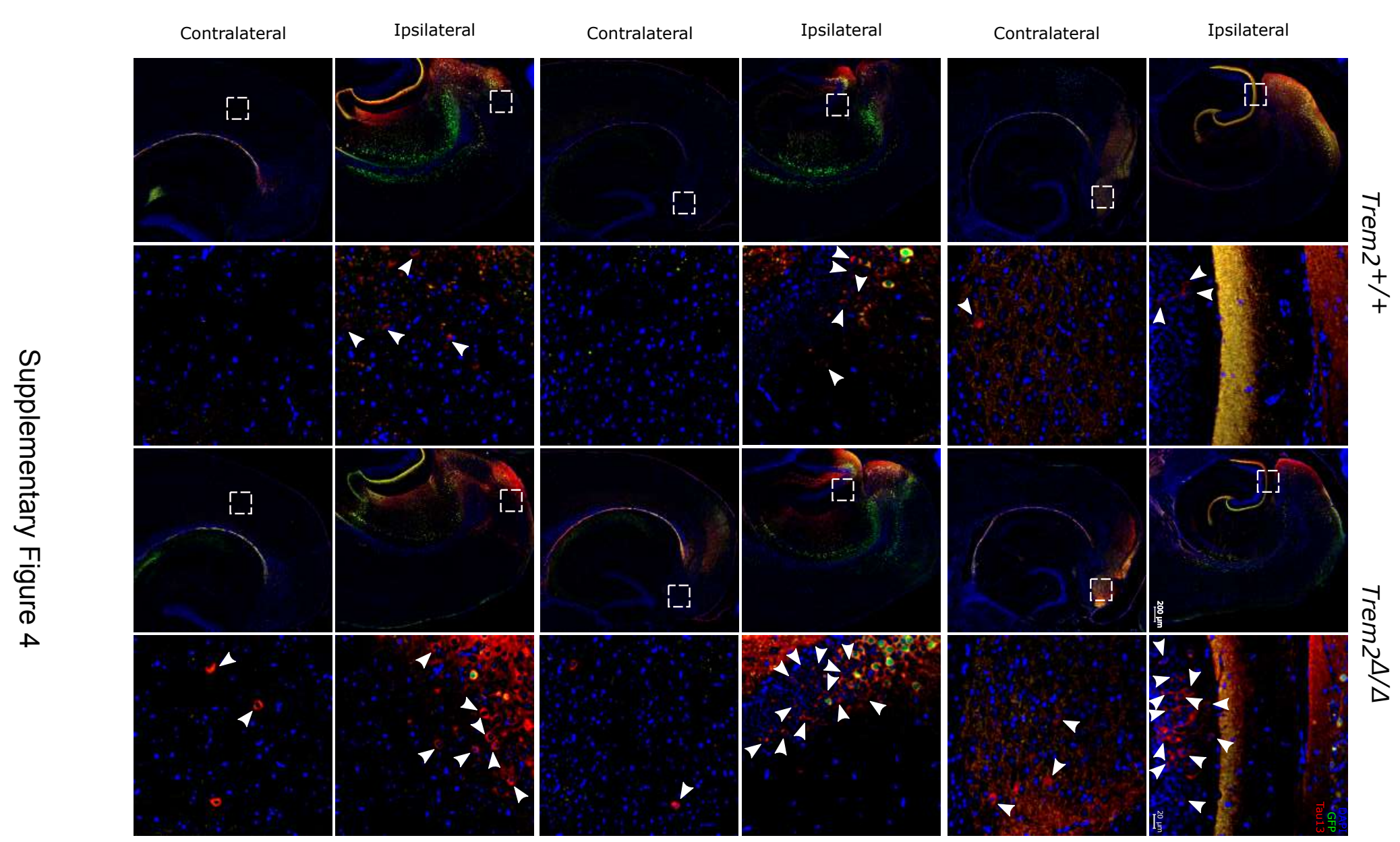


Table S1

Animals used in behaviour tests

\begin{tabular}{|c|c|c|c|c|c|c|c|c|c|c|c|c|c|c|c|c|c|c|}
\hline \multirow[t]{2}{*}{ Genotype } & Test & \multicolumn{4}{|c|}{$\mathbf{R R}$} & \multicolumn{4}{|c|}{ Beam } & \multicolumn{4}{|c|}{ EPM } & \multicolumn{4}{|c|}{ OF } & \multirow{2}{*}{$\begin{array}{c}\text { MWM } \\
3\end{array}$} \\
\hline & \begin{tabular}{l|l}
$\mathrm{N}$ & Age (months)
\end{tabular} & 1 & 2 & 3 & 6 & 1 & 2 & 3 & 6 & 1 & 2 & 3 & 6 & 1 & 2 & 3 & 6 & \\
\hline \multirow{3}{*}{$\begin{array}{l}\text { TAU58/ } \\
\text { Trem2 }^{\Delta / \Delta}\end{array}$} & total & 10 & 12 & 10 & 11 & 10 & 12 & 9 & 6 & 9 & 10 & 7 & 8 & 10 & 10 & 10 & 11 & 10 \\
\hline & $M$ & 5 & 6 & 5 & 9 & 5 & 6 & 4 & 4 & 5 & 7 & 4 & 2 & 5 & 6 & 5 & 9 & 5 \\
\hline & $\mathrm{F}$ & 5 & 6 & 5 & 2 & 5 & 6 & 5 & 2 & 4 & 3 & 3 & 6 & 5 & 4 & 5 & 2 & 5 \\
\hline \multirow[t]{3}{*}{ Trem2 $2^{\Delta / \Delta}$} & total & 11 & 10 & 11 & 14 & 11 & 10 & 11 & 8 & 11 & 10 & 11 & 10 & 11 & 10 & 10 & 14 & 10 \\
\hline & M & 4 & 4 & 5 & 9 & 4 & 4 & 5 & 5 & 4 & 4 & 5 & 7 & 4 & 4 & 5 & 9 & 5 \\
\hline & $F$ & 7 & 6 & 6 & 5 & 7 & 6 & 6 & 3 & 7 & 6 & 6 & 3 & 7 & 6 & 5 & 5 & 5 \\
\hline \multirow{3}{*}{$\begin{array}{l}\text { TAU58/ } \\
\text { Trem2 }^{\Delta /+}\end{array}$} & total & 14 & 13 & 13 & 12 & 14 & 12 & 10 & 3 & 14 & 12 & 10 & 7 & 14 & 9 & 13 & 12 & 11 \\
\hline & $M$ & 8 & 11 & 7 & 8 & 8 & 10 & 4 & 1 & 8 & 10 & 4 & 5 & 8 & 8 & 7 & 8 & 6 \\
\hline & $\mathrm{F}$ & 6 & 2 & 6 & 4 & 6 & 2 & 6 & 2 & 6 & 2 & 6 & 2 & 6 & 1 & 6 & 4 & 5 \\
\hline \multirow[t]{3}{*}{ Trem2 $2^{\Delta /+}$} & total & 13 & 13 & 10 & 29 & 13 & 13 & 10 & $\overline{16}$ & 12 & 12 & 8 & 20 & 13 & 12 & 10 & 29 & 8 \\
\hline & $M$ & 6 & 4 & 4 & 20 & 6 & 4 & 4 & 14 & 6 & 3 & 3 & 16 & 6 & 3 & 4 & 20 & 2 \\
\hline & $\mathrm{F}$ & 7 & 9 & 6 & 9 & 7 & 9 & 6 & 2 & 6 & 9 & 5 & 4 & 7 & 9 & 6 & 9 & 6 \\
\hline \multirow{3}{*}{$\begin{array}{l}\text { TAU58/ } \\
\text { Trem2 }^{+/+}\end{array}$} & total & 10 & 10 & 11 & 12 & 10 & 10 & 11 & 7 & 10 & 9 & 10 & 9 & 10 & 9 & 11 & 12 & 10 \\
\hline & $M$ & 2 & 4 & 3 & 5 & 2 & 4 & 3 & 1 & 2 & 4 & 2 & 4 & 2 & 4 & 3 & 5 & 5 \\
\hline & $\mathrm{F}$ & 8 & 6 & 8 & 7 & 8 & 6 & 8 & 6 & 8 & 5 & 8 & 5 & 8 & 5 & 8 & 7 & 5 \\
\hline \multirow[t]{3}{*}{ Trem2 $2^{+/+}$} & total & 11 & 11 & 19 & 16 & 11 & 11 & 19 & 7 & 11 & 11 & 15 & 13 & 11 & 11 & 19 & 15 & 14 \\
\hline & $M$ & 4 & 5 & 8 & 9 & 4 & 5 & 8 & 3 & 4 & 5 & 5 & 7 & 4 & 5 & 8 & 9 & 6 \\
\hline & $\mathrm{F}$ & 7 & 6 & 11 & 7 & 7 & 6 & 11 & 7 & 7 & 6 & 10 & 6 & 7 & 6 & 11 & 6 & 8 \\
\hline
\end{tabular}


Table S2

Antibodies used for histology

\begin{tabular}{|l|l|l|}
\hline Antibody & Company & Dilution \\
\hline pS214 & Abcam & $1 / 1000$ \\
\hline pS422 & Abcam & $1 / 500$ \\
\hline Gfap & Sigma & $1 / 200$ \\
\hline Iba-1 & Wako & $1 / 250$ \\
\hline Tau13 & Abcam & $1 / 500$ \\
\hline PHF-1 & gift from P. Davies & $1 / 250$ \\
\hline GFP rbt & Abcam & $1 / 100$ \\
\hline GFP goat & Abcam & $1 / 200$ \\
\hline DAPI & Molecular Probes & $1 / 1000$ \\
\hline AlexaFluor mse 555 & Invitrogen & $1 / 250$ \\
\hline AlexaFluor rbt 488 & Invitrogen & $1 / 250$ \\
\hline AlexaFluor rbt 647 & Invitrogen & $1 / 250$ \\
\hline
\end{tabular}


Table S3

Primers list

\begin{tabular}{|l|l|l|}
\hline Gene name & Forward & Reverse \\
\hline Siglech & AAGAGTTGGTCCAGGGAAACC & GCCTGATCCATGTGTGTTGC \\
\hline CD16 & CCCTGATATCAAGTCGCGCA & TTCTCAAGCAGCGAGACAGG \\
\hline C4 & CAGGACAGAACAGTGGAGCA & ATCTTCAGCACGAAGGCAGT \\
\hline ApoE & CTCCCAAGTCACACAAGAACTG & CCAGCTCCTTTTTGTAAGCCTTT \\
\hline Trem2 & ACAGCACCTCCAGGAATCAAG & ACTTGCTCAGGAGAACGCAG \\
\hline
\end{tabular}


Table S4

Antibody panel CyTOF

\begin{tabular}{|c|c|c|c|c|c|}
\hline Antigen & Metals & Clone & Vendor & Cell type & Description \\
\hline \multicolumn{6}{|c|}{ Lineage markers } \\
\hline B220 & $\mathrm{Nd} 144$ & RA3.3A1 & Fluidigm & B cells, pDC & lineage/ signal transduction in hematopoiesis \\
\hline Ly6G & Pr141 & $1 \mathrm{~A} 8$ & Fluidigm & neutrophils, eosinophil subset & migration \\
\hline Siglec-H & Gd158 & 551 & BioLegend & microglica, pDC & reduces type I IFN response \\
\hline CD45.2 & Sm147 & 30-F11 & Fluidigm & pan-leukocytes & TCR and BCR signal transduction complex \\
\hline CD11b & Nd148 & $\mathrm{M} 1 / 70$ & Fluidigm & myeloid cells, NK cells & complement receptor 3 \\
\hline Ly6C & Nd150 & HK1.4 & Fluidigm & myeloid cells & urokinase-type plasminogen activator receptor (UPAR) \\
\hline CD3 & Sm152 & $145-2 C 11$ & Fluidigm & T cells & T cell co-receptor \\
\hline Ter119 & Sm154 & TER-119 & Fluidigm & erythrocytes & glcophorin-A associated protein \\
\hline Siglec- $\mathrm{F}$ & $\mathrm{Nd} 145$ & E50-2440 & BD & eosinophiles, alveolar macrophages & cell adhesion \\
\hline CD64 & Eu151 & $\mathrm{X} 54-5 / 7.1$ & Fluidigm & macrophages, monocytes & Igh affiity IgG receptor \\
\hline NK1.1 & Ho165 & PK136 & Fluidigm & NK cells & CD161, killer cell lectin-like receptor \\
\hline CD11c & $\mathrm{Bi} 209$ & N418 & Fluidigm & DC & cell - cell contact \\
\hline $\mathrm{F} 4 / 80$ & Tb159 & BM8 & Fluidigm & macrophages, microglia & cell adhesion, cell activation \\
\hline CD117 & Er166 & 2B8 & Fluidigm & stem cells, lymphocytes & c-kit \\
\hline \multicolumn{6}{|c|}{ Immune modulation } \\
\hline CD88 & Gd160 & $20 / 70$ & BioLegend & granulocytes, monocytes, macrophages & complement receptor 5 \\
\hline CD14 & Gd156 & Sa14-2 & Fluidigm & macrophages & LPS co-receptor \\
\hline Sca-1 & Tm169 & D7 & Fluidigm & stem cells, lymphocytes & stem cell antigen 1 \\
\hline CD206 & Er168 & $\mathrm{CO} 8 \mathrm{C} 2$ & BioLegend & macrophages, DC & PAM \\
\hline C3aR & Dy161 & 14D4 & HyCult & myeloid and microglia & complement receptor \\
\hline CD36 & $\mathrm{Nd} 146$ & HM36 & BioLegend & monocytes & scavanger receptor \\
\hline \multicolumn{6}{|c|}{ Migration \& Differentiation } \\
\hline CCR2 & Dy163 & 475301 & R\&D Systems & monocytes, macrophages, immature B cells & CCL2 receptor, anti-APC \\
\hline CX3CR1 & Dy164 & SA011F11 & Fluidigm & macrophages, monocytes & migration \\
\hline CD16/32 & Er167 & 93 & BioLegend & myeloid cells, Nk cells & low affinioty IgG receptor \\
\hline PD-L1 & Eu153 & 10F.9G2 & BioXcell & multiple cells & co-inhibitor, activated cells \\
\hline CD44 & Yb171 & IM7 & Fluidigm & most cell types, memory and activated T cells & homing, adhesion \\
\hline CD86 & Yb172 & GL-1 & Fluidigm & antigen presenting cells & co-stimulation ligand \\
\hline CD172 & Yb173 & P84 & BioLegend & myeloid cells, stem cells, neurons & SIRP1a \\
\hline
\end{tabular}




\begin{tabular}{|c|c|c|c|c|c|}
\hline MHC2 & Yb174 & M5/114.15.2 & Fluidigm & antigen presenting cells & antigen presentation \\
\hline CD38 & Lu175 & 90 & Fluidigm & $\begin{array}{l}\text { macrophages, B cells, monocytes, lymphocytes, } \\
\text { neurons }\end{array}$ & metabolism, differentiation \\
\hline FcER1a & Yb176 & Mar-01 & Fluidigm & mast cells, basophils, monocytes, DC & High affinity IgE receptor \\
\hline CD163 & Er170 & S150491 & BioLegend & myeloid cells, microglia & scavanger receptor \\
\hline \multicolumn{6}{|c|}{ Microglia } \\
\hline MerTK & $\mathrm{Nd} 143$ & polyclonal & R\&D Systems & macrophages & multifunctional, anti-biotin \\
\hline P2X7R & Nd142 & $1 F 11$ & BioLegend & macrophages, microglia & binding of extracellular adenosine 5 '-triphosphate (ATP) \\
\hline P2Y12R & Dy162 & S16007D & BioLegend & macrophages, microglia & binding of extracellular adenosine 5'-triphosphate (ATP) \\
\hline
\end{tabular}

\section{Other reagents CyTOF}

\begin{tabular}{|c|c|c|c|c|c|}
\hline Term & Metals & Target & Vendor & Cell type & Description \\
\hline BC106 & Cd106 & CD45.2 (104.2) & BioXcell & all immune cells and microglia & technical-barcoding reagents \\
\hline $\mathrm{BC} 110$ & Cd110 & CD45.2 (104.2) & BioXcell & all immune cells and microglia & technical-barcoding reagents \\
\hline BC111 & Cd111 & CD45.2 (104.2) & BioXcell & all immune cells and microglia & technical-barcoding reagents \\
\hline BC112 & Cd112 & CD45.2 (104.2) & BioXcell & all immune cells and microglia & technical-barcoding reagents \\
\hline BC113 & $\mathrm{Cd} 113$ & CD45.2 (104.2) & BioXcell & all immune cells and microglia & technical-barcoding reagents \\
\hline BC114 & Cd114 & CD45.2 (104.2) & BioXcell & all immune cells and microglia & technical-barcoding reagents \\
\hline BC116 & Cd116 & CD45.2 (104.2) & BioXcell & all immune cells and microglia & technical-barcoding reagents \\
\hline dead & Rh103 & DNA, extracellular & Fluidigm & all cells & technical-live/dead stain \\
\hline DNA1 & Ir191 & DNA, intracellular & Fuidigm & all cells & technical-cellular maker \\
\hline DNA2 & Ir193 & DNA, intracellular & Fluidigm & all cells & technical-cellular maker \\
\hline
\end{tabular}

